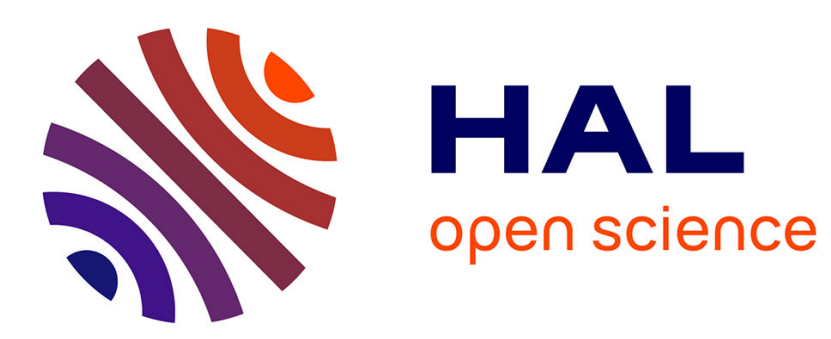

\title{
Aerodynamic behavior of a floating offshore wind turbine
}

Caroline Lienard, Ronan Boisard, Camille Daudin

\section{To cite this version:}

Caroline Lienard, Ronan Boisard, Camille Daudin. Aerodynamic behavior of a floating offshore wind turbine. AIAA Scitech 2019, Jan 2019, SAN DIEGO, United States. hal-02088810

\section{HAL Id: hal-02088810 https://hal.science/hal-02088810}

Submitted on 3 Apr 2019

HAL is a multi-disciplinary open access archive for the deposit and dissemination of scientific research documents, whether they are published or not. The documents may come from teaching and research institutions in France or abroad, or from public or private research centers.
L'archive ouverte pluridisciplinaire HAL, est destinée au dépôt et à la diffusion de documents scientifiques de niveau recherche, publiés ou non, émanant des établissements d'enseignement et de recherche français ou étrangers, des laboratoires publics ou privés. 


\title{
Aerodynamic behavior of a floating offshore wind turbine
}

\author{
Lienard C., ${ }^{1}$ Boisard R. ${ }^{2}$, Daudin C. ${ }^{3}$ \\ ONERA - The French Aerospace Lab \\ F-92190 Meudon, France
}

\begin{abstract}
The flowfield around the rotor blades of a wind turbine may be quite complex due to the occurrence of several aerodynamic phenomena. It is all the more true for floating offshore wind turbines (FOWT), for which the six rigid-body motions of the floating platform can induce blade/wake interactions. Therefore conventional numerical approaches for wind turbine applications, such as BEM, may be questionable for an accurate prediction of floating wind turbine aerodynamic loads. Consequently, the current paper investigates the aerodynamic behavior of a FOWT subjected to several prescribed motions, representative of a wave movement, based on CFD simulations. These results, obtained on the NREL 5-MW wind turbine, are compared to previous results found in the literature and analyzed to provide a better understanding of the involved aerodynamic phenomena.
\end{abstract}

\section{Nomenclature}

$\begin{array}{ll}a & =\text { shaft angle }\left(^{\circ}\right) \\ \delta & =\text { precone angle }\left({ }^{\circ}\right) \\ \mathrm{R} & =\text { NREL } 5 \text {-MW blade radius }(\mathrm{m}) \\ A_{\text {PITCH }} & =\text { pitch motion amplitude } \\ f_{\text {PITCH }} & =\text { pitch motion frequency } \\ \theta & =\text { pitch angle } \\ A_{\text {SURGE }} & =\text { surge motion amplitude } \\ f_{\text {SURGE }} & =\text { surge motion frequency } \\ d x & =\text { surge displacement } \\ C_{P} & =\text { pressure coefficient }\end{array}$

\section{Introduction}

In 2015, within the framework of the COP21 meeting held in Paris, France has committed itself to cover 32\% of its energy demand with renewable energy by 2030 . To do so, France benefits from a privileged location for marine renewable energies to emerge and develop, with its $3500 \mathrm{~km}$ of coastline considering metropolitan areas only. Offshore wind turbines are so far the preferred solution, since they are currently technologically more advanced than water power or tidal power technologies. However, if fixed offshore wind turbines are already widely installed in several countries, floating offshore wind turbines (FOWT) are still in the prototype stage. They consist in an attractive alternative compared to fixed wind turbines in order to move them away from the coasts. They are nevertheless also exposed to more severe environmental conditions, and particularly to the wave movement.

The interest in FOWT being quite recent, the scientific literature dealing with this topic develops progressively: Sebastian and Lackner [1], Tran et al. [2][3][4], Leble et al. [5], Bekiropoulos et al. [6], Cheng et al. [7] and Li et al. [8] employ a variety of numerical methodologies to characterize the aerodynamics of these FOWT, while experimental results obtained on FOWT remain quite scarce because of the cost of the needed facilities. The PhD thesis of Courbois from Centrale Nantes [9] can however be mentioned with an experimental investigation of a FOWT under wind and wave loads.

To investigate the wind turbine aerodynamic behavior, ONERA can rely on two different numerical tools with different fidelity level, already widely used and validated on aeronautical applications: PUMA as a fast-response

\footnotetext{
${ }^{1}$ Research Scientist, Aerodynamics, Aeroelasticity, Acoustics Department (DAAA).

${ }^{2}$ Research Scientist, Aerodynamics, Aeroelasticity, Acoustics Department (DAAA).

${ }^{3}$ Intern, Aerodynamics, Aeroelasticity, Acoustics Department (DAAA).
} 
approach, and elsA as a high fidelity code. These tools have already been tested and validated on the MEXICO wind turbine in the framework of the IEA Task 29 project MexNextIII [10], the New MEXICO experimental database being used to perform a code to code comparison between all the participants.

The current paper aims at quantifying and analyzing the effect of several prescribed motions on the aerodynamic performances of a floating wind turbine, and will mainly rely on elsA CFD results. Some PUMA results can however be punctually used in order to have access to information which are more easily extracted from lifting line approaches.

\section{Floating wind turbine geometry and associated motions}

The selected wind turbine for this investigation is the NREL 5-MW wind turbine, designed by the National Renewable Energy Laboratory to be a representative utility-scale wind turbine suitable for a floating offshore installation [11]. It is a conventional three-bladed upwind turbine, with a rotor diameter of $126 \mathrm{~m}$. The rotor blades are composed of series of DU (Delft University) and NACA 64xxx airfoils from the hub to the tip. The mast is 90 meters high but it should be noted that the mast will not be taken into account in the present simulations. All the details about the NREL 5-MW wind turbine can be found in [11]. The more significant parameters and properties are summarized in Table 1 below.

Table 1 - Design parameters of the NREL 5-MW wind turbine

\begin{tabular}{|cc}
\hline Rating & $5 \mathrm{MW}$ \\
\hline Rotor orientation, configuration & Upwind, 3 blades \\
Rotor, hub diameter & $126 \mathrm{~m}, 3 \mathrm{~m}$ \\
Hub height & $90 \mathrm{~m}$ \\
\hline Cut-in, rated, cut-out wind speed & $3 \mathrm{~m} \cdot \mathrm{s}^{-1}, 11.4 \mathrm{~m} \cdot \mathrm{s}^{-1}, 25 \mathrm{~m} . \mathrm{s}^{-1}$ \\
Cut-in, rated rotation regime & $6.9 \mathrm{rpm}, 12.1 \mathrm{rpm}$ \\
Rated blade tip speed & $80 \mathrm{~m} \cdot \mathrm{s}^{-1}$ \\
Shaft tilt, precone & $5^{\circ}, 2.5^{\circ}$ \\
\hline
\end{tabular}

Once the wind turbine geometry chosen, a bibliographic review helped to define which motion to prescribe to the wind turbine, both representative of the floating behavior and quite simple to implement. The platform floating motion indeed includes six degrees of freedom, three translational and three rotational components (Fig. 1):

- The heave motion, which is the vertical translational movement;

- $\quad$ The sway motion, for the lateral translational movement;

- The surge motion, which is a translation in the axial direction;

- The yaw motion, a rotation around the vertical axis;

- $\quad$ The pitch motion, which is the rotational movement around the lateral axis;

- $\quad$ The roll motion, for the rotation around the axial axis.

Sebastian and Lackner [1] deeply investigated these 6 DOFs and characterized the modes which result in unsteady aerodynamic phenomena, making aerodynamic analysis of offshore floating wind turbines a challenge. Their main conclusions, based on FAST simulations, are that "Surge and pitch DOFs appear to be the largest amplitudes modes" for all the simulated platforms, leading them to be chosen for the current investigation.

These motions can possibly amplify existing unsteady aerodynamic phenomena, such as blade-vortex interaction and dynamic stall, or induce unsteady effects due to wind shear, gradient across the rotor disk, skewed flow and rapid local velocity modifications. Figure 2 notably emphasizes to which extent the flowfield around the wind turbine during a pitching motion becomes highly complex and unsteady: as the rotor begins to pitch back, it can interact with its own wake, leading to the development of recirculation areas. This explains why most design methods, usually based on the blade element momentum theory, can not accurately predict the aerodynamic performances and behavior of FOWT: unsteady numerical tools, "of higher-fidelity 'engineering-level' models than commonly in use" [1] are needed to model these effects. 


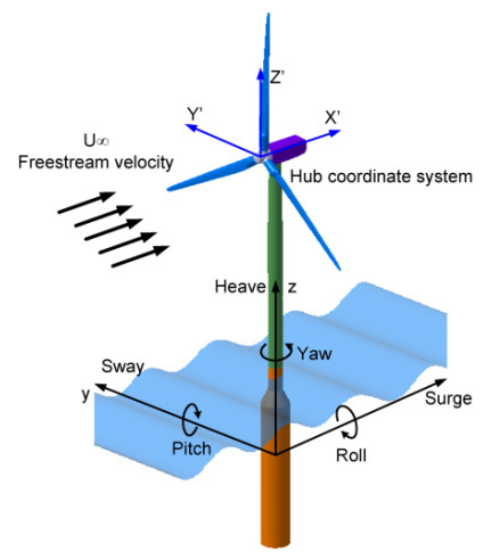

Fig. 1 Degrees of freedom for an offshore floating wind turbine (from [2])

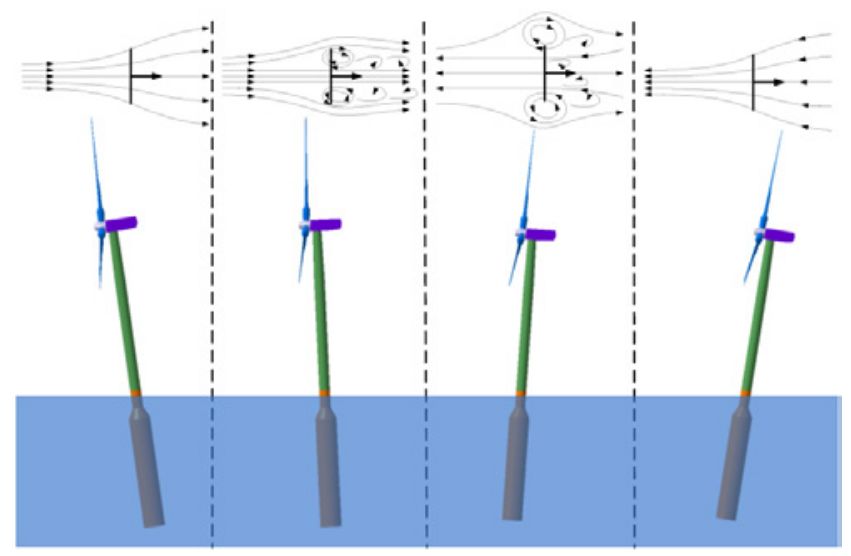

Fig. 2 Platform pitching motion and its effect on the surrounding flowfield (from [12])

\section{Numerical approaches}

As already mentioned, two different levels of numerical simulation are used at ONERA on wind turbines: a fastresponse one, based on the lifting line theory coupled to a free wake model, and a high fidelity one, based on unsteady CFD computations. The current investigation relies on complex time-accurate CFD simulations but the results may be complemented when necessary by free-wake information (local angle of attack or velocities for example).

\section{A. Lifting line/Free-Wake approach}

The PUMA (Potential Unsteady Methods for Aerodynamics) code, which is developed at ONERA since 2013, is based on a long lasting knowledge about free wakes methods for helicopter aerodynamics. It is built on a coupling between an aerodynamic module and a kinematic module. The aerodynamic module relies on a free wake model and a lifting line approach. The free wake model is based on Mudry theory [13] which rigorously describes the unsteady evolution of a wake modeled by a potential discontinuity surface. The lifting line method relies on 2D airfoils characteristics and can handle some 3D corrections for blade sweep and 2D unsteady aerodynamics effects through dynamics stall models. Moreover, different time discretizations are available in order to balance between accuracy, scheme stability and computational time. Concerning the kinematic module, it is based on a rigid multi-body system approach using a tree-like structure with links and articulations. In order to reduce computational time, the code has been parallelized using OpenMP and the Multilevel Fast Multipole Method has been implemented for the computation of the velocities induced by each wake panel on any element. PUMA is extensively used at ONERA for any aerodynamic study which requires low computational cost or a large amount of parametrical investigation like pre-design studies. It has been successfully applied on quite a lot of different fixed wings and rotating wings configurations like propellers, counter rotating open rotors, helicopter rotors [14] and more recently wind turbines [10].

\section{B. CFD approach}

The CFD computations are performed using elsA CFD package, co-owned by Airbus, Safran and ONERA [15][16]. This package contains a RANS flow solver and several external modules, especially pre- and postprocessing tools (Cassiopee tools [17]). elsA is dedicated to the numerical simulation of the compressible viscous mono-species steady and unsteady flows, on three-dimensional (or two-dimensional, or axisymmetric) multi-block structured meshes. It solves the compressible (U)RANS equations in a finite volume cell-centered formulation. The mesh strategy is based on the Chimera approach: a curvilinear body-fitted grid is built around each component, which are assembled and embedded into a set of Cartesian background grids.

The wind turbine model includes the blades, a rotating hub and a fixed rear nacelle (Fig. 3), the influence of the mast being neglected. When the pitch and the surge motions are implemented, the whole turbine is moving in the 
fixed background grid. The grid is quite refined, with a total of about 162 million points: approximately 7.5 million points per blade (Fig. 4), 3.5 million points for the nacelle and the hub, 132 million points in the background grids. In order to correctly model and convect the blade tip vortices, the smallest cell size in the Cartesian background grid is equivalent to $10 \%$ of the mean chord of the blade. Moreover, so as to enable good wake conservation even when the wind turbine is moving, this finest background grid level extends far beyond the wind turbine (up to $2.25 R$ ), and also upstream (Fig. 4).

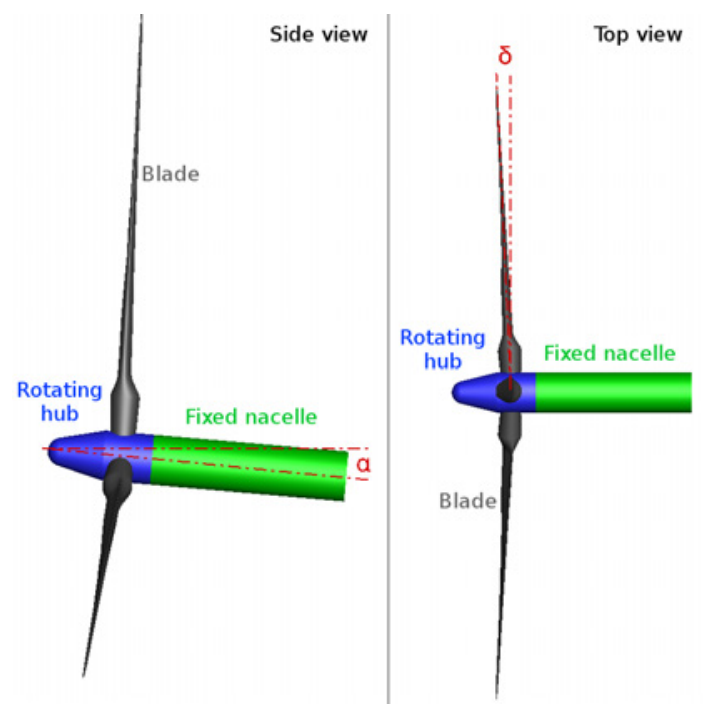

Fig. 3 NREL 5-MW parts considered for the CFD simulations
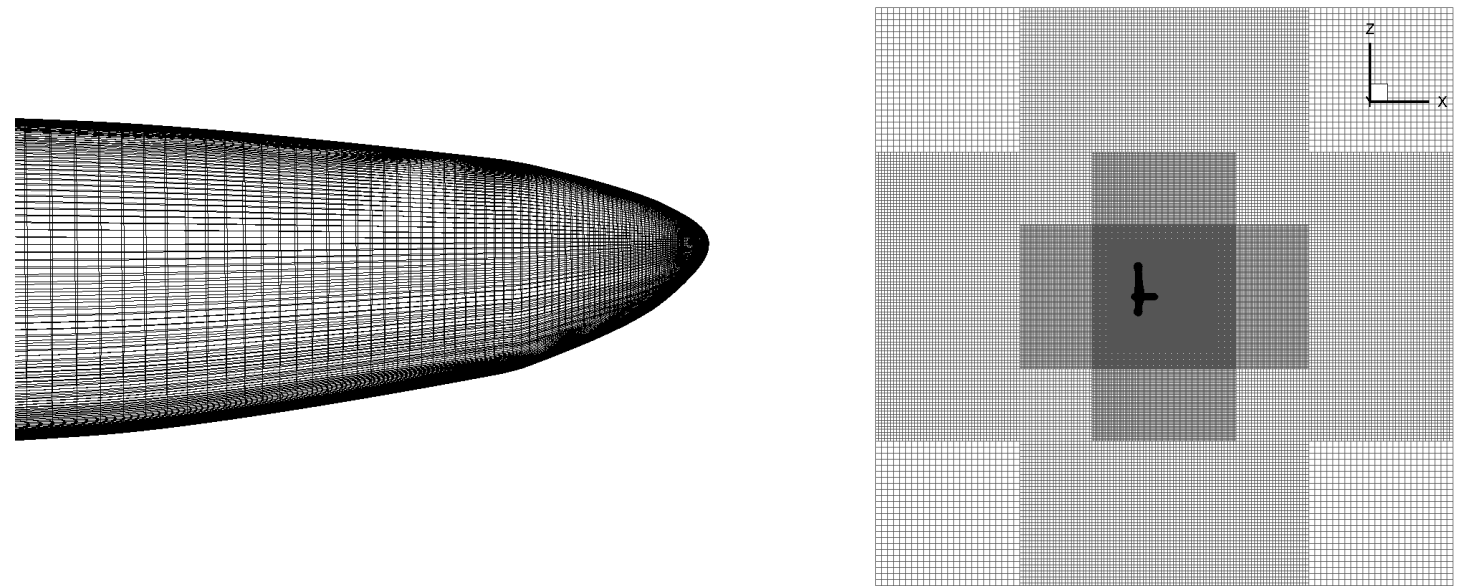

Fig. 4 Blade tip surface mesh (left) and wind turbine in the Cartesian background grid (right)

All these simulations are performed using the $2^{\text {nd }}$ order AUSM+P spatial scheme (chosen for its low dissipation and its low-Mach capabilities), with a time integration ensured by a $2^{\text {nd }}$ order implicit backward finite difference scheme solved by a Newton algorithm. In order to ensure good accuracy, the number of Newton sub-iterations was set to 25. A physical timestep corresponding to an azimuthal angle of $1^{\circ}$ was used for the first revolutions and then refined up to $0.5^{\circ}$. The chosen turbulence model is $k-\omega$ Kok. All CFD computations consider a freestream velocity of $11.4 \mathrm{~m} \cdot \mathrm{s}^{-1}$ and a constant rotating speed of $12.1 \mathrm{rpm}$ (rated freestream velocity and rotation regime). This rotation speed, as well as the blade pitch angle, is fixed for all computations.

These CFD simulations are obviously extremely expensive, with a computational time of about 27000 CPU hours per rotor revolution with a $0.5^{\circ}$ timestep, corresponding to a 96-144 hours restitution time on 300 Nehalem-EP $(2.8 \mathrm{GHz})$ processors. At least 16 rotor revolutions are necessary to reach sufficient convergence. Most of these computations were performed on the HPC resources of CINES under the allocation 2018 - A0032A10280 made by GENCI. 


\section{Validation on the fixed NREL 5-MW wind turbine}

Both PUMA end elsA simulations have first been performed on the baseline case of the fixed NREL 5-MW wind turbine. They have enabled to define some numerical parameters, especially for the free-wake approach, and have been validated against the thrust and power curves provided by the NREL and obtained with FAST code (Fig. 5). CFD results have also been compared to the one obtained by Kirby et al [18] (Fig. 6).

Fig. 5 reveals that the power curve is very well reproduced by PUMA simulations, especially up to the rated regime. More discrepancies are observed on the thrust curve between FAST and PUMA even if the global trend is very similar. These discrepancies may be partly explained by the fact that FAST takes into account the blade deformation whereas both PUMA and elsA simulations consider rigid blades. The CFD results obtained with els $A$ at the nominal operating point are in very good agreement with FAST (Fig. 6): the thrust is 3.2 \% lower than in FAST results, whereas the power is $2.4 \%$ higher. It has to be noted that 16 rotor revolutions have been computed to obtain these results, with a timestep of $0.5^{\circ}$ on the last 9 revolutions. After the first 7 rotor revolutions with a timestep of $1^{\circ}$, the obtained power was indeed overestimated by $6.3 \%$ compared to FAST (Fig. 7).

Consequently, both methods (PUMA and elsA simulations) seem to be validated on this fixed wind turbine case with regard to performance evaluation.

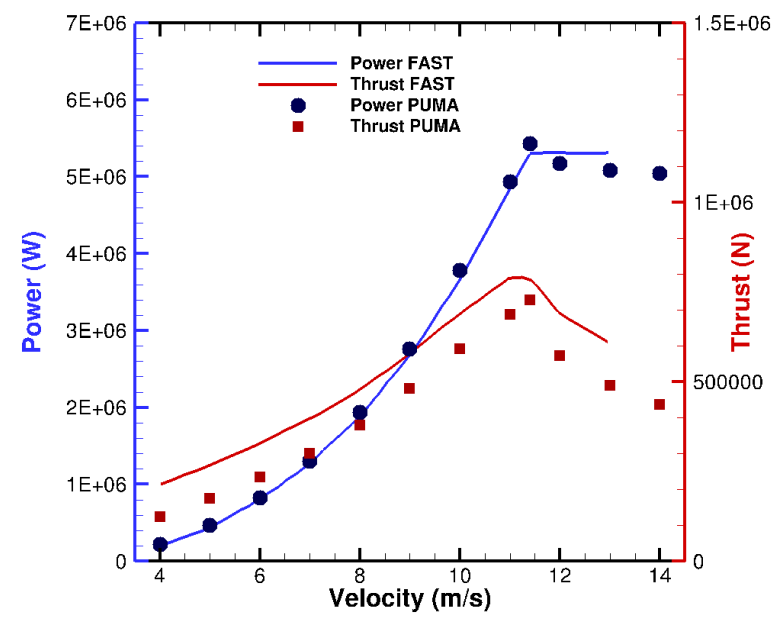

Fig. 5 Fixed WT: Power and thrust comparison between FAST and PUMA

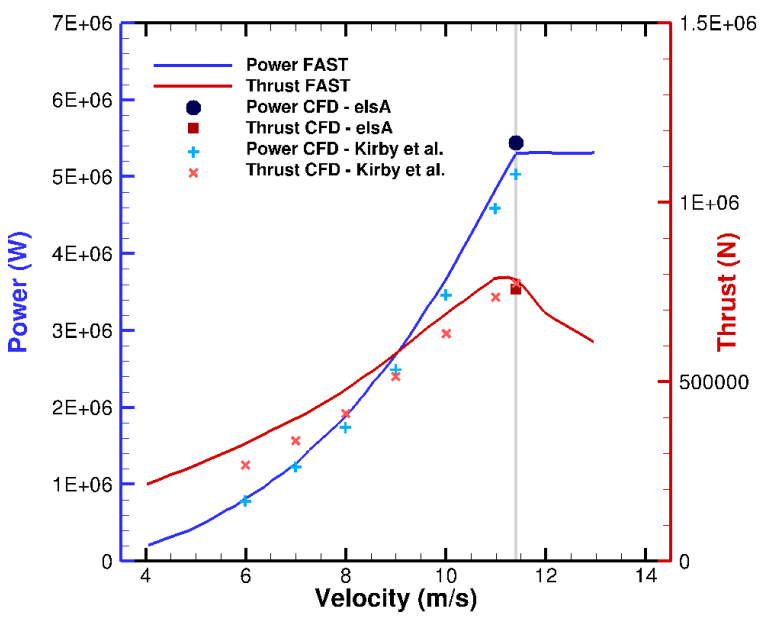

Fig. 6 Fixed WT: Power and thrust comparison between FAST, elsA and CFD results obtained by

Kirby et al. [18]

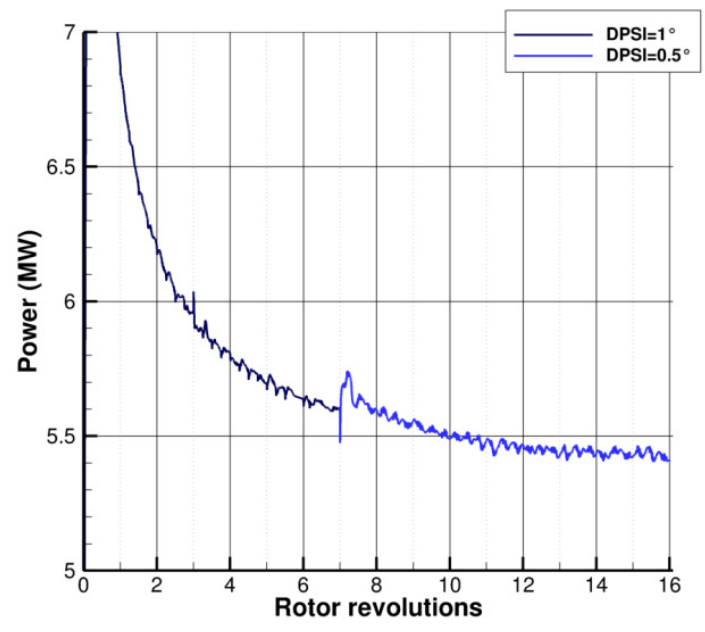

Fig. 7 Fixed WT: Power convergence from elsA simulation 
Furthermore, the wake downstream the wind tunnel was shown to be well captured and convected without being too much dissipated (thanks to the AUSM+P scheme and to the extended finest background grid level). Fig. 8 indeed emphasizes that 3 rotor revolutions are kept in the wake, which will be of great interest to investigate blade/wake interactions once the FOWT motion will be taken into account.
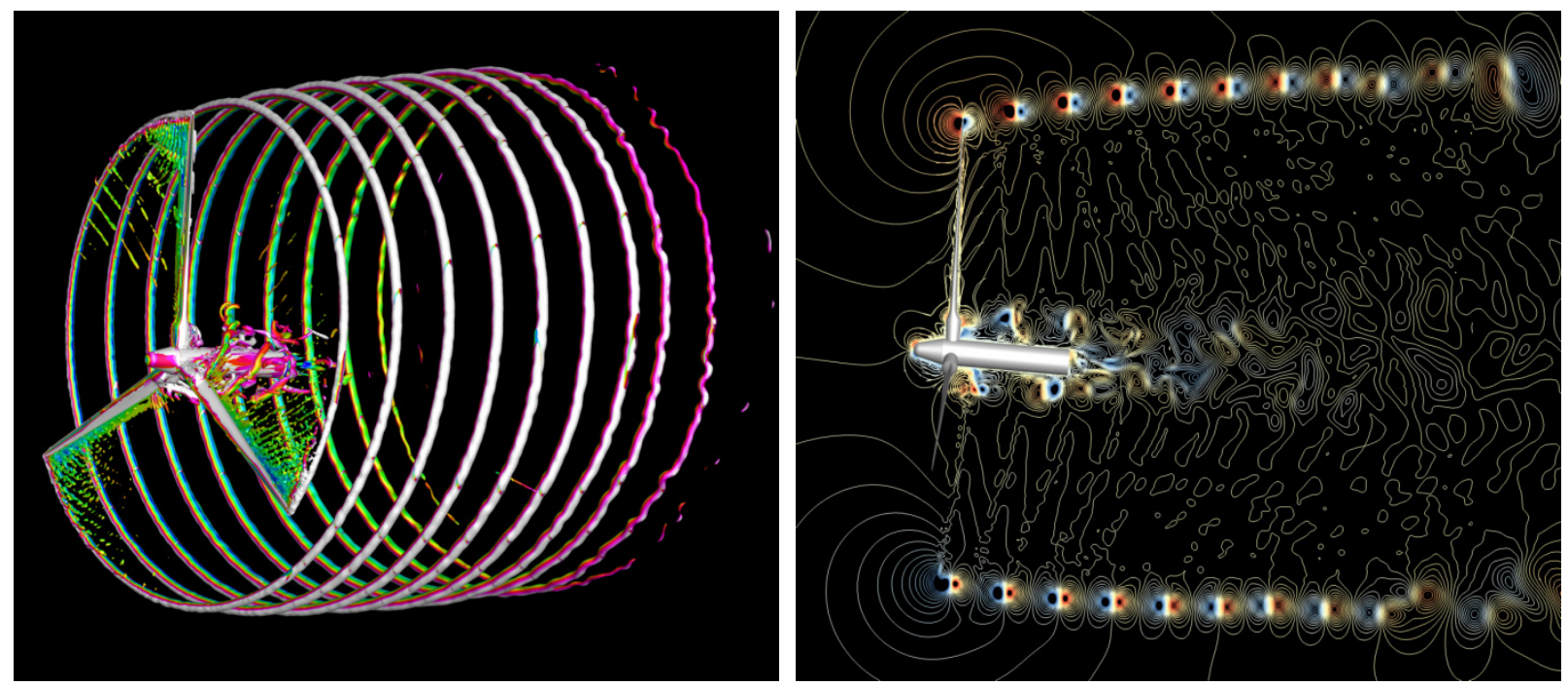

Fig. 8 Fixed WT: Q-criterion isosurface (left) and velocity in the symmetry plane (right) from elsA simulation

\section{Simulation of the floating offshore wind turbine: pitch motion}

\section{A. Motion description}

The pitch motion of the FOWT in elsA computations consists in a prescribed motion applied to the whole wind turbine grids (except the background grids), defined as follows:

$$
\theta(t)=A_{\text {PITCH }} * \sin \left(2 \pi f_{\text {PITCH }} t\right)
$$

$A_{\text {PITCH }}$ refers to the pitch motion amplitude and $f_{\text {PITCH }}$ to the pitch motion frequency. Several amplitudes have been simulated, including $4^{\circ}$ and $8^{\circ}$, whereas the frequency was decided to be kept to half the blade rotation frequency (ie. approximately $0.1 \mathrm{~Hz}$ ). This was motivated by the fact that it would ease the post processing and the analysis to have a periodic movement, and this value was quite similar to what was found in the literature. The same motion has been easily implemented in PUMA simulations since it only consists in an additional articulation in the tree-like

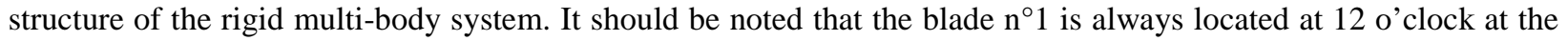
beginning of the motion, which starts in the backward direction.

Fig. 9 is a representation of the pitch motion (for $A_{P I T C H}=4^{\circ}$ ) and should help to analyze the upcoming results. The wind turbine pitch angle is plotted over two pitching periods (4 rotor revolutions) in red (a positive pitch angle corresponding to a backward position), as well as the pitch angular velocity in green. One can already notice that the maximum angular velocity in absolute terms is obtained when the wind turbine comes back to its initial position $\left(\mathrm{T}_{0}\right.$ and $\mathrm{T}_{4}$, corresponding to $\theta(t)=0^{\circ}$ ). The inflow velocity normal to the rotor plane (at the hub center) is finally added in grey and defined as follows:

$$
V_{\text {norm }}=V_{W I N D} * \cos (\theta)-\dot{\theta} * h_{M A S T}
$$

The maximum normal inflow velocity is obtained at $\mathrm{T}_{4}$, when the wind turbine comes back to its initial position in a forward motion. 


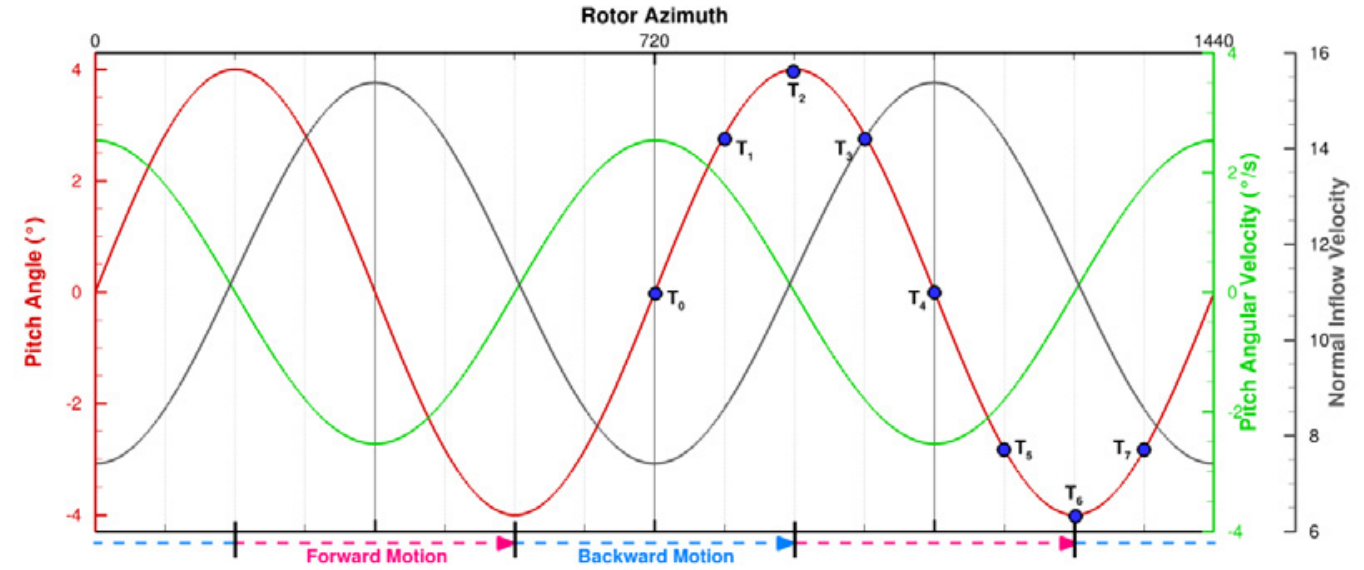

Fig. 9 Pitch motion analysis (for $A_{P I T C H}=4^{\circ}$ and $f_{P I T C H} \approx 0.1 \mathrm{~Hz}$ ): WT pitch angle (red), WT pitch angular velocity (green) and relative axial velocity (grey)

\section{B. Wind turbine loads}

The results obtained in terms of global thrust and power for both amplitudes are plotted in Fig. 10 and Fig. 11.
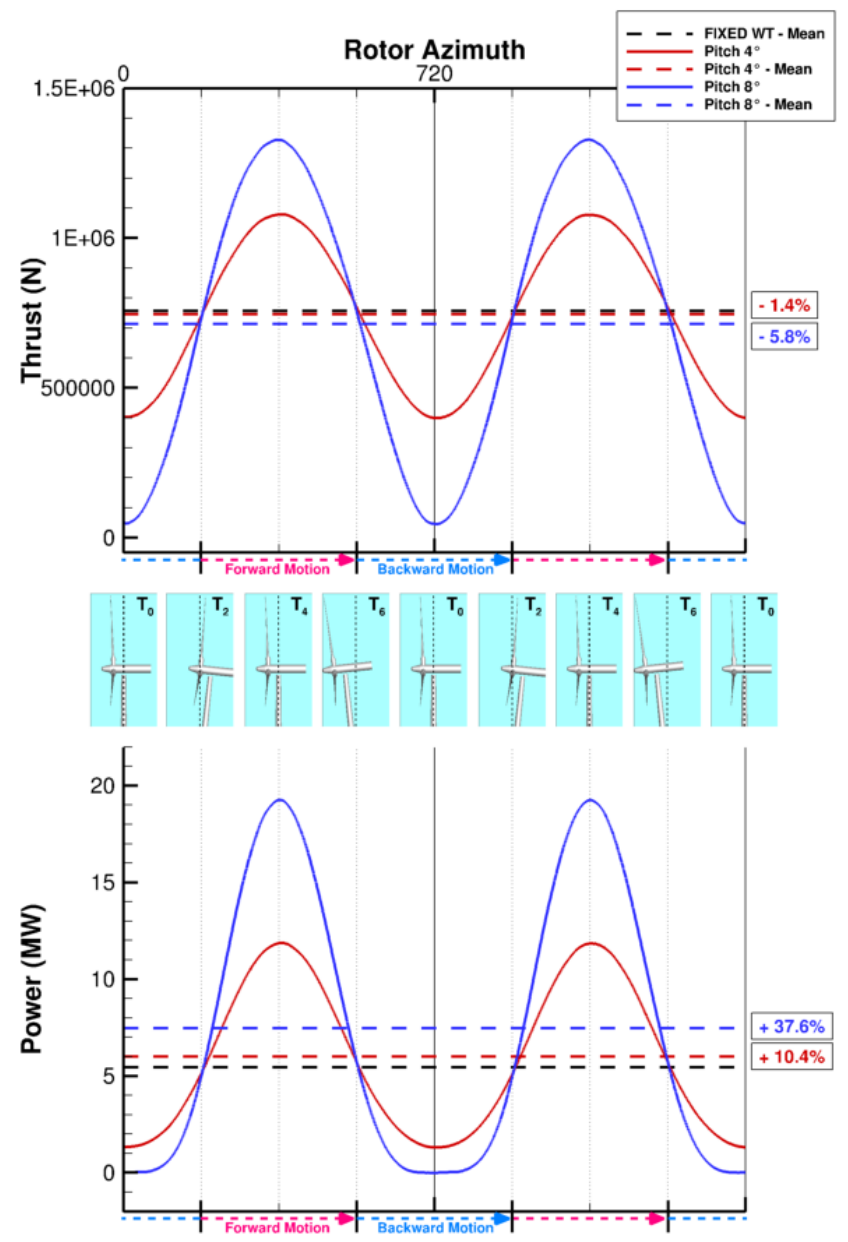

Fig. 10 Pitch motion: Global thrust and power as function of time
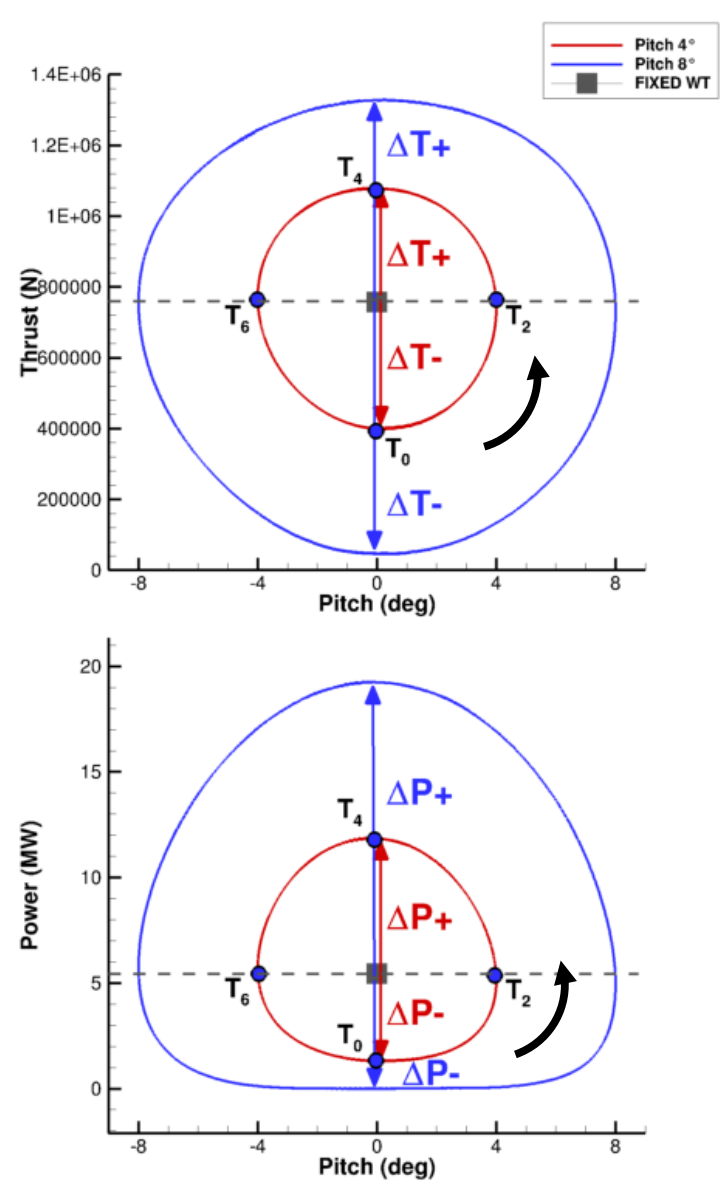

Fig. 11 Pitch motion: Global thrust and power as function of pitch angle 
On the one hand, in Fig. 10, the global thrust and power are plotted as a function of time over 2 pitching periods. The respective mean values are added and compared to the fixed wind turbine case. Although the results show large variations of both thrust and power, one can notice that the mean thrust is only slightly impacted by the pitch motion: it is decreased by $1.4 \%$ for $A_{P I T C H}=4^{\circ}$ and by $5.8 \%$ for $A_{P I T C H}=8^{\circ}$. However, the difference is significantly larger when looking at the power curves: the power of the wind turbine is increased by $10.4 \%$ for $A_{\text {PITCH }}=4^{\circ}$ and by $37.6 \%$ for $A_{P I T C H}=8^{\circ}$. The wind turbine undergoing a pitch motion with a $4^{\circ}$-amplitude (which is quite realistic) hence experiences instantaneous aerodynamic power variations from almost 0MW to 12MW. These results are very close to those obtained on the same wind turbine in [4] and on the DTU 10MW wind turbine in [5]. It is also very clear that the power curve maximum and minimum seen in Fig. 10 are linked to the normal inflow velocity extrema presented in Fig. 9 respectively at $\mathrm{T}_{4}$ and $\mathrm{T}_{0}$.

On the other hand, Fig. 11 shows the obtained thrust and power as functions of the pitch angle, forming a hysteresis loop. This plot first highlights for both amplitudes that $\Delta P+>\Delta P-$ and that $\Delta T+<\Delta T-:$ over one pitching period, the wind turbine power is increased whereas the thrust is decreased. This is mainly due to the fact that the power hysteresis loop is flattened in its lower part, that is to say between $T_{6}$ and $T_{2}$. This portion of the curve corresponds to the backward motion phase, which induces the lowest normal inflow velocities on the rotor plane (cf. Fig. 9).

Obviously, the same behavior is visible when looking at the power curves on each blade (Fig. 12): when the pitch amplitude reaches $8^{\circ}$, the aerodynamic power of each blade during the backward motion phase becomes very low, reaching slightly negative values between $T_{6}$ and $T_{0}$ for blades 1 and 2 , and between $T_{0}$ and $T_{2}$ for blades 1 and 3. However the global power remains positive because of the third blade. One should keep in mind that a real wind turbine would not reach negative power values, the wind turbine would probably slow down. The prescribed constant rotation speed is responsible for this and is a limitation on such computations.

Fig. 12 also emphasizes that the loads variations are more important on blade 1: this is due to the fact that blade 1 is located at 12 o'clock at the beginning of the motion, inducing a higher lever arm with respect to the pitching motion center compared to the other blades.

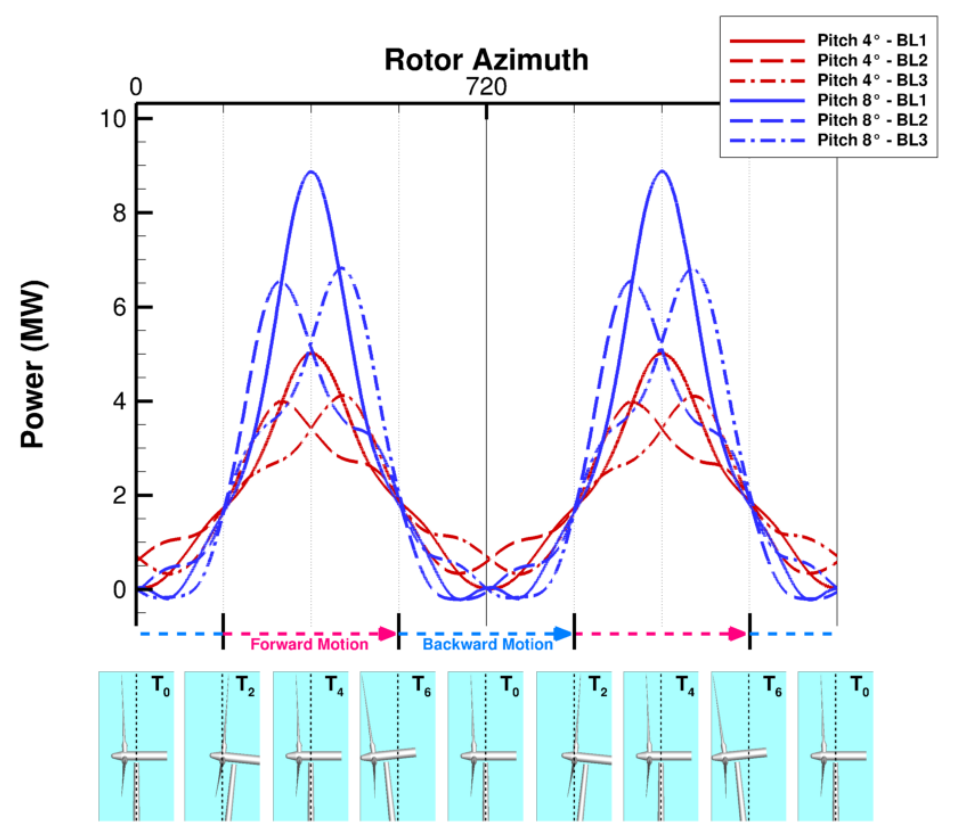

Fig. 12 Pitch motion: Blade power variations as function of time

It can also be interesting to look at what happens locally on each blade. To do so, PUMA simulations have been performed in addition to CFD: it is indeed much easier to get local blade section velocities and angles of attack from a lifting-line/free-wake approach than from a CFD result. Moreover, PUMA and elsA simulations have provided comparable results in terms of loads and loads variations on the baseline case. Thus, Fig. 13 depicts the local blade section velocity magnitude variations due to the pitch motion, and Fig. 14 displays the local blade section angle of attack for the $4^{\circ}$-amplitude pitch case. These two plots are very similar to the loads sectional representations which 
are commonly used on helicopter rotors. However, two rotor revolutions have to be plotted in order to have one pitch motion period.

Even if the fluctuations of inflow velocity normal to the rotor plane are rather important (of the order of $50 \%$ ), Fig. 13 reveals that the local blade section velocity variations due to the motion are quite limited on the three blades and mainly located near the blades root. On such configurations, the local inflow is indeed dominated by the rotational velocity. However, this modification of the normal inflow velocity has a significant impact on the blade section angle of attack, as seen on the scheme in Fig. 15. This is confirmed by the PUMA results obtained for a $4^{\circ}$ pitch motion in Fig. 14: strong variations of the local angle of attack can be seen on all blades. While this angle of attack remains close to $+5^{\circ}$ on the fixed wind turbine case, the pitch motion induces variations between $-2^{\circ}$ (during the backward motion phase) to approximately $+10^{\circ}$ (during the forward motion phase).
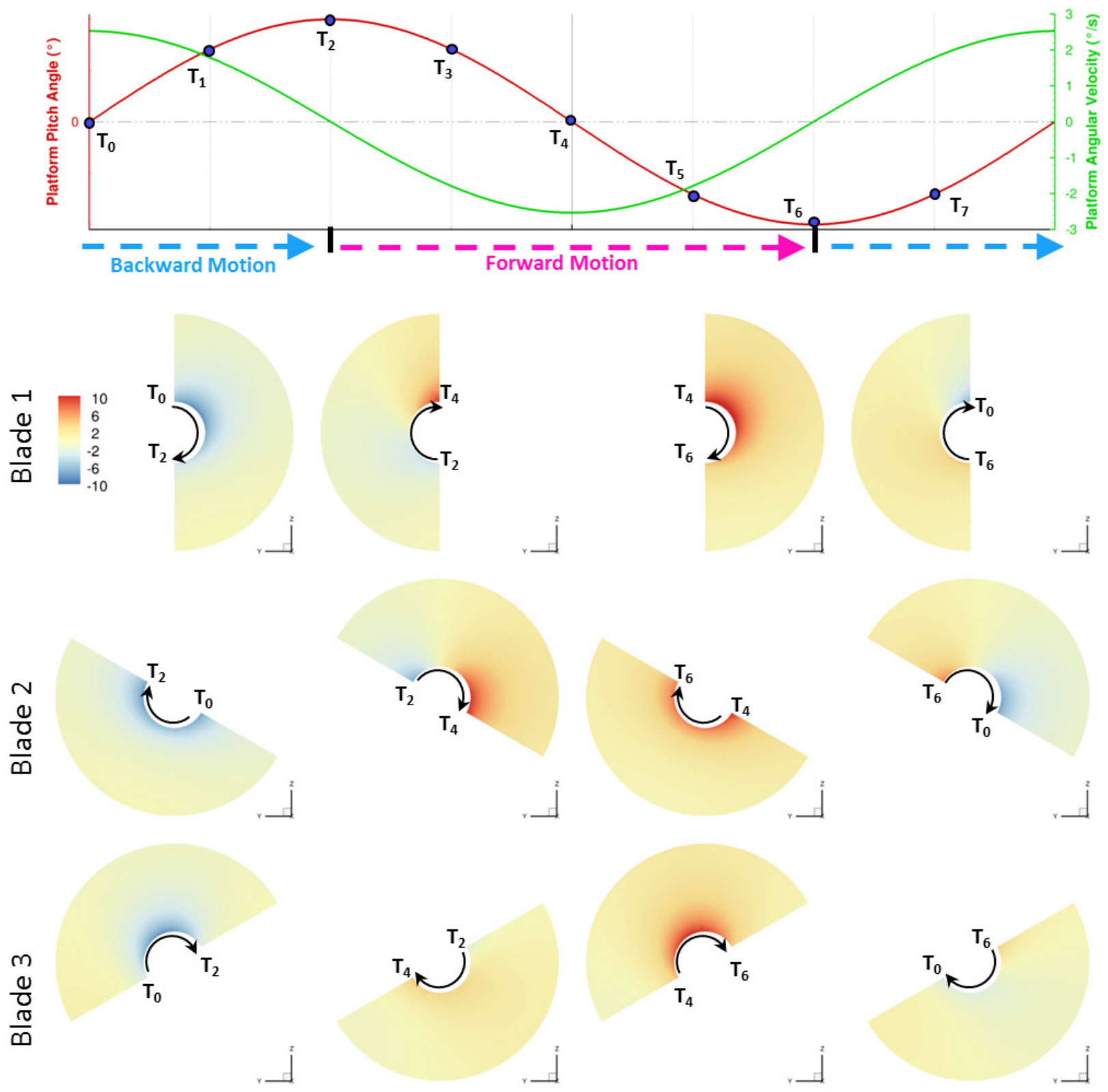

Fig. 13 Pitch motion: Local blade section velocity magnitude variations due to the pitch motion with $A_{P I T C H}=$ $4^{\circ}$ (in \%) 

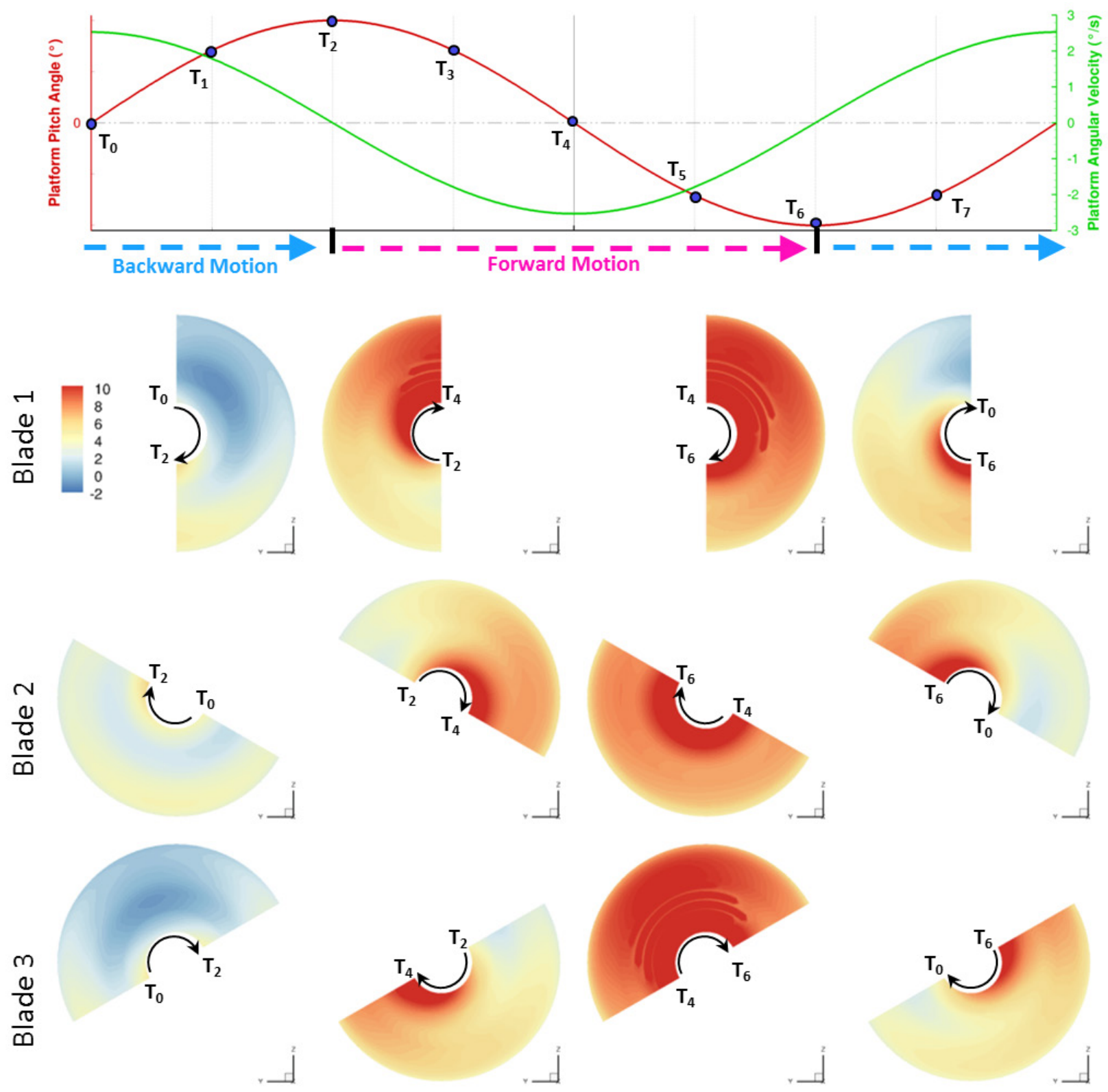

Fig. 14 Pitch motion: Local blade section angle of attack for the $4^{\circ}$-amplitude test case

When the pitch amplitude is increased to $8^{\circ}$ for the same frequency, the fluctuations of normal inflow velocity are obviously larger, leading to larger variations of local angles of attack (from $-6^{\circ}$ to $+19^{\circ}$ ). In such conditions, the wind turbine is operating out of its nominal design conditions, which could lead to very low (or even negative) values of aerodynamic power. For example, the NACA64 airfoil characteristics are presented in Fig. 16 with the angle of attack variations obtained for both amplitudes pitch motion at $75 \%$ radius. One can notice that this airfoil operates close to zero lift and close to stall conditions when $A_{P I T C H}=4^{\circ}$, and negative lift and real stall conditions are reached when the amplitude is increased to $8^{\circ}$. This behavior explains the very low power values obtained during the backward motion of the wind turbine. Moreover, the global power increase obtained during the forward motion will be limited by blade stall if the motion amplitude or frequency are increased. 


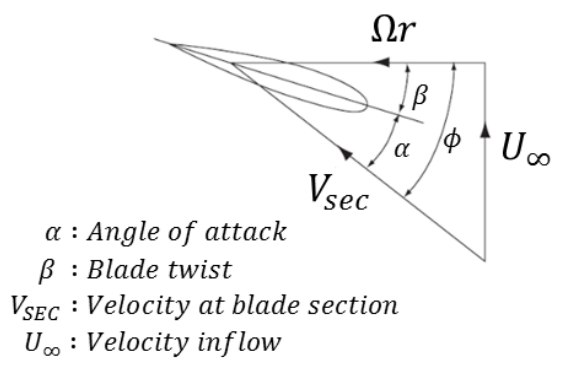

Fig. 15 Local blade section velocity scheme

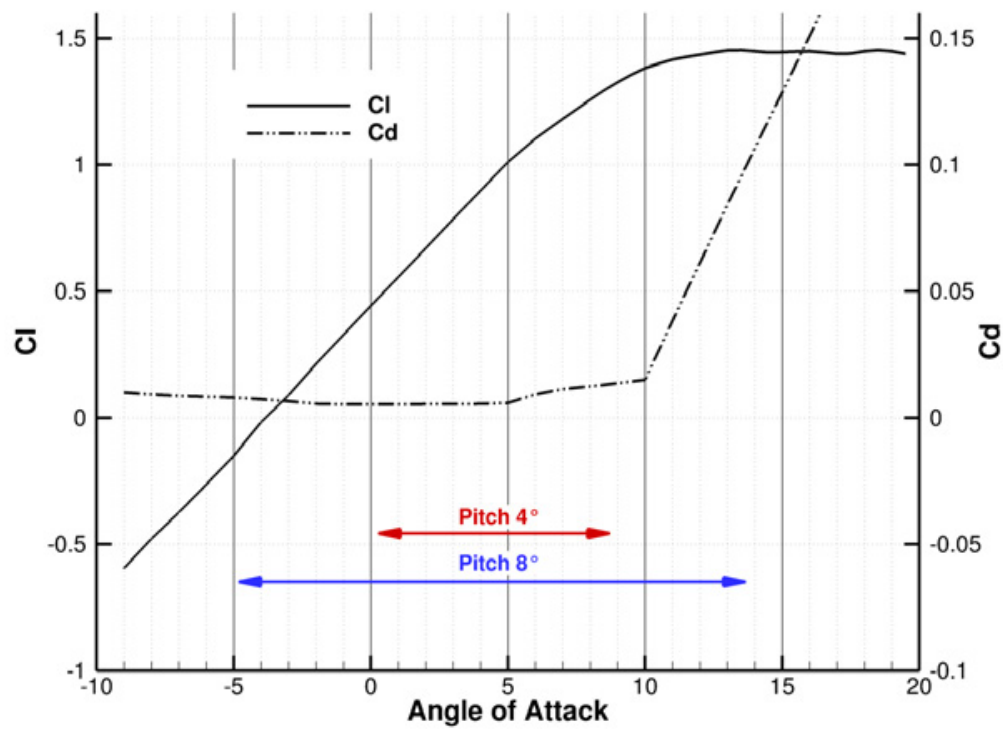

Fig. 16 NACA64 airfoil characteristics

\section{Blade spanwise loads}

The mean blade spanwise loads obtained for the three cases (fixed WT, $A_{P I T C H}=4^{\circ}$ and $A_{P I T C H}=8^{\circ}$ ) are plotted in Fig. 17. The mean spanwise thrust and power are unsurprisingly almost identical on the three blades for the fixed WT: only slight differences can be seen because of the shaft angle. However these discrepancies are negligible compared to the ones obtained for the two pitch motion cases.

For both thrust and power distributions, the first blade, which is located at 12 o'clock at the beginning of the motion, differs from the two others: this blade $n^{\circ} 1$ produces less thrust and more power. This result is consistent with the results presented in Fig. 12.
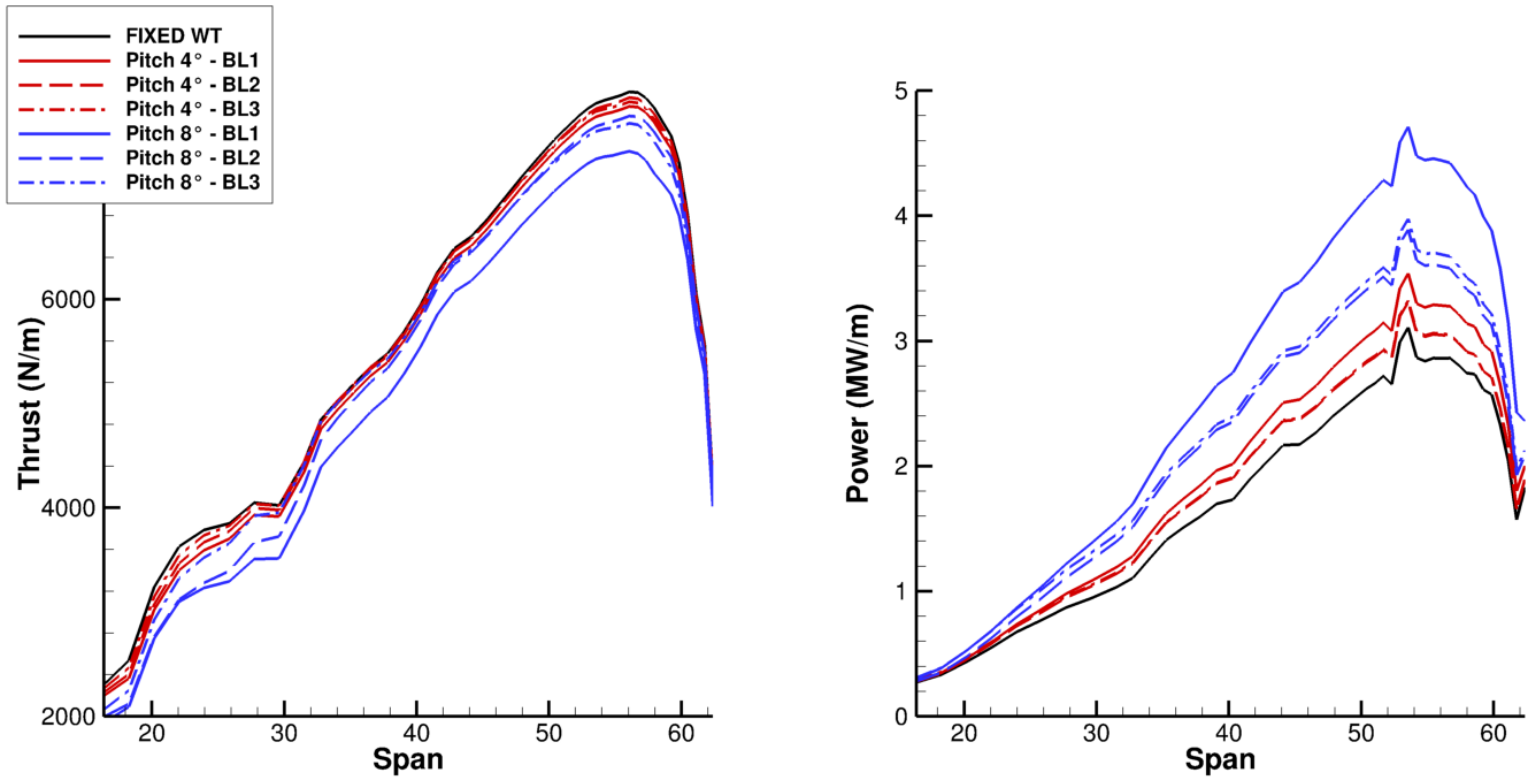

Fig. 17 Pitch motion: Mean spanwise loads on the 3 blades compared to the fixed WT case 
Fig. 18 focuses on the $4^{\circ}$-amplitude case by representing the mean thrust and power distributions together with their respective envelope (maximum and minimum values). This figure demonstrates that the loads variations on blade $\mathrm{n}^{\circ} 1$ are also higher than on the two other blades. These discrepancies between the blades in terms of mean loads and load variations can be a big issue on a real wind turbine regarding blades deformation, vibrations, fatigue,... It should however be kept in mind that this behavior is overestimated in the CFD simulations because of the wind turbine and motion frequencies synchronization along with the prescribed constant rotation speed.
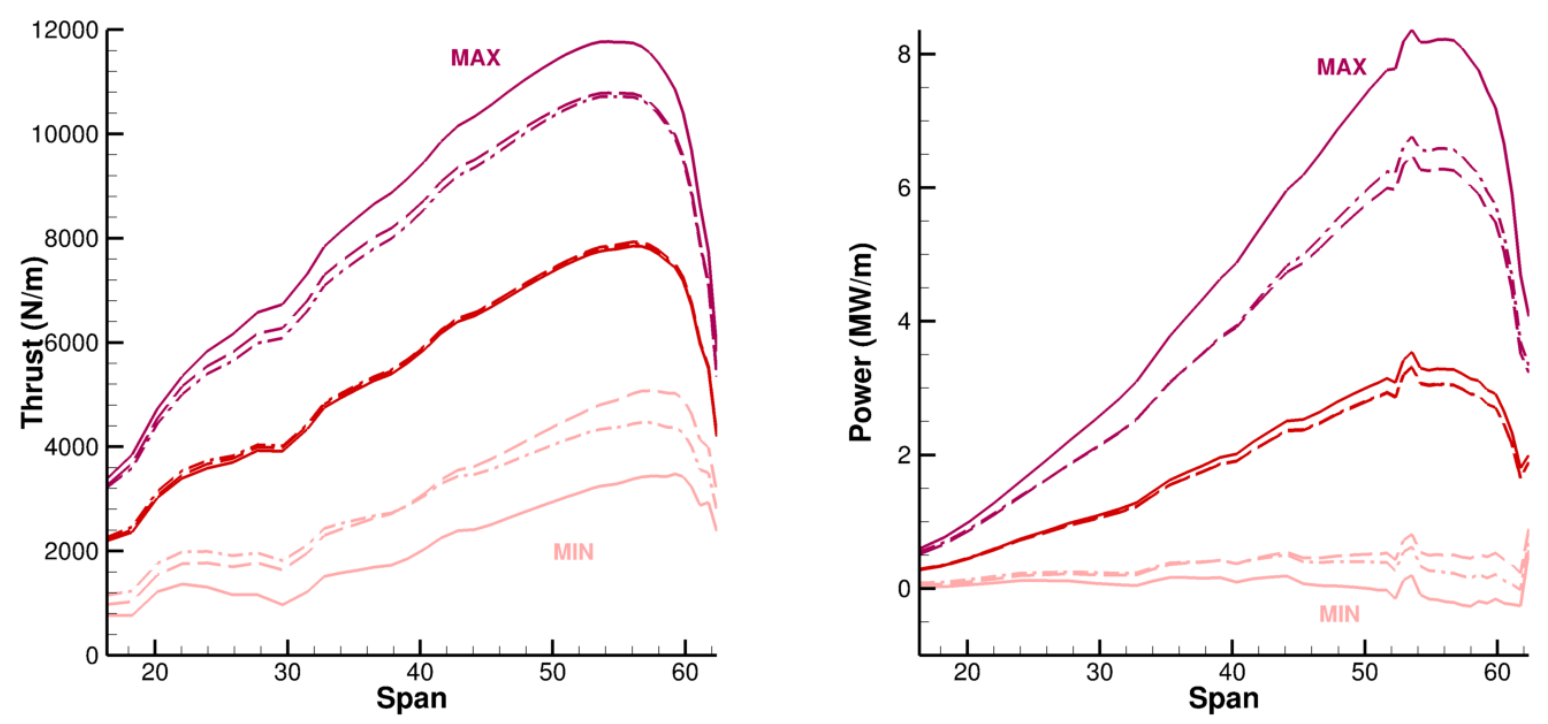

Fig. 18 Pitch motion: Mean spanwise loads and loads envelope on the 3 blades for $A_{P I T C H}=4^{\circ}$

\section{Wake visualizations}

For both pitching amplitude, the wake of the wind turbine at several timesteps is shown in Fig. 19 and Fig. 20 thanks to q-criterion isosurfaces and velocity contours in the symmetry plane. These two representations help to follow the vortices trajectory and to identify the interactions between vortices or between the wind turbine and its own wake. The presented timesteps correspond to the markers $\left(T_{0}, T_{1}, T_{2}, \ldots\right)$ defined in Fig. 9, where a positive pitch angle corresponds to a rear position.

First, one can notice that the wake is really disrupted for the $8^{\circ}$-amplitude case. This amplitude is probably excessive compared to what can happen on a real FOWT but the idea was to amplify the motion so as to confirm the physical phenomena observed on the $4^{\circ}$ case.

Then, on both cases, the simulations meet the expectations in terms of wake conservation since several interaction phenomena seem to be visible: blade/vortex interactions, vortex pairing...

In order to help analyzing these interactions, a new figure is produced based on helicopter reasoning and on Leble and Barakos investigation on FOWT aerodynamic [5]. When studying helicopters in descending flight, the ratio between the normal velocity to the rotor plane (here $V_{c}=-V_{\text {norm }}$, where the negative sign is added to agree with helicopter notations) and a "theoretical" induced velocity in hover for a given thrust $\left(V_{h}\right)$ is often plotted to identify VRS (Vortex Ring State) conditions [19]. The same approach is used in Fig. 21 (based on [5]) with $V_{h}=\sqrt{T / 2 \rho S}$, T being the mean thrust obtained on a pitching period and $\mathrm{S}$ the rotor area. Vortex Ring State conditions would be reached for $-1 \leq V_{c} / V_{h} \leq 0$ (thus approximately when the wind turbine moves beyond its wake).

When looking at the wake visualizations for $A_{\text {PITCH }}=4^{\circ}$, some blade/vortex interactions seem to appear, especially in Fig. 19a): the new tip vortex is emitted very close to the previous one due to the maximum pitch angular velocity at $T_{0}$. These interactions actually do not occur for the maximum pitch angle of the wind turbine $\left(\mathrm{T}_{2}\right)$ but for the minimum inflow velocity normal to the rotor plane. The wind turbine is not truly operating in VRS since the blade is not immersed in the wind turbine wake, which is confirmed by Fig. 21. The wake is however in a 
turbulent state with very strong interactions between blade tip vortices. Some vortex pairing phenomena can be seen in Fig. 19, producing wake meandering and leading to instabilities in the flow downstream the wind turbine.

For the second pitch case $\left(A_{\text {PITCH }}=8^{\circ}\right)$, Fig. 21 seem to reveal that the rotor partially enters VRS between $T_{7}$ and $\mathrm{T}_{0}$. This is confirmed by Fig. 20a), where the wind turbine has moved beyond its own wake, with the new tip vortex clearly emitted downstream the preceding blade one. These two vortices then roll-up together (Fig. 20c)) and are convected faster in the wake compared to the other vortices (from Fig. 20d) to Fig. 20h)). Once again, this results in wake meandering and strong instabilities downstream the FOWT. It has to be noted as well that these interactions rather occur on the top of the wind turbine: this is due to the shaft angle on the one hand, and to the local axial velocity which is different between the top and the bottom of the wind turbine.

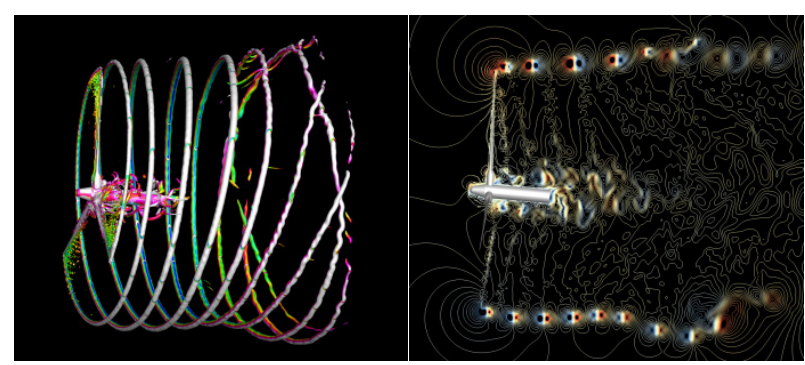

a) $\mathbf{T}_{\mathbf{0}}$

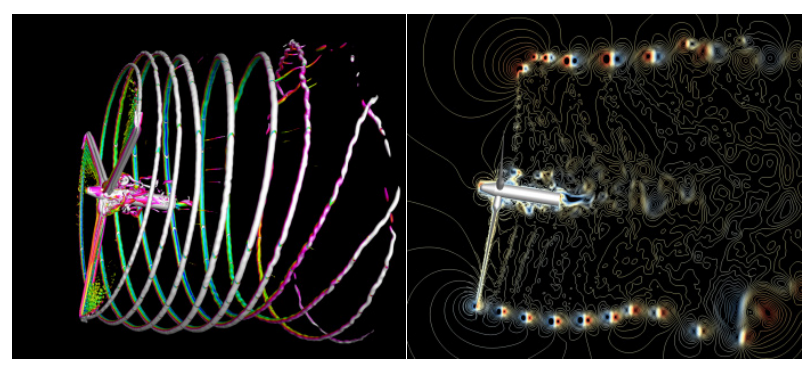

c) $\mathbf{T}_{2}$

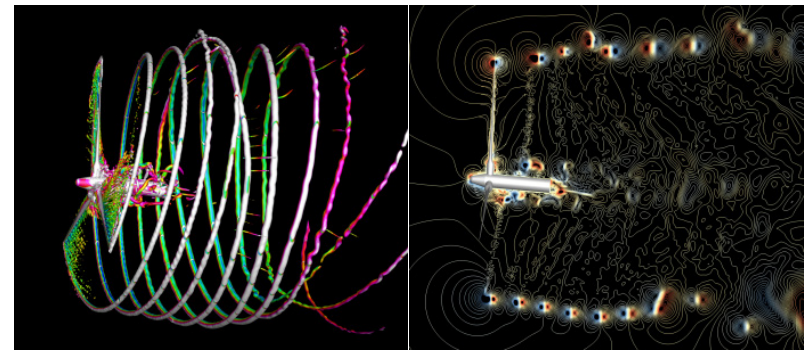

e) $\mathbf{T}_{4}$

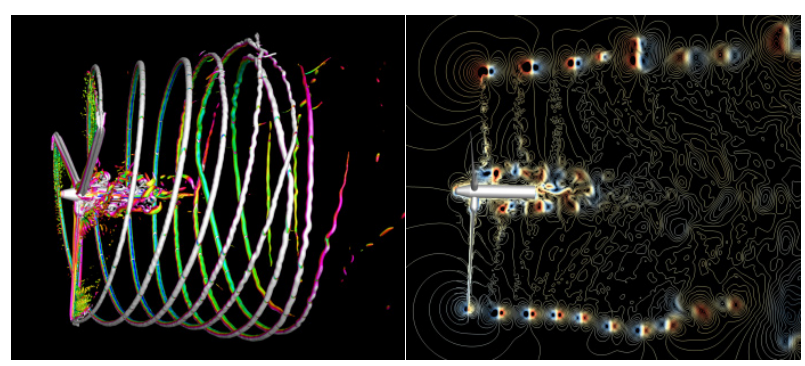

g) $T_{6}$

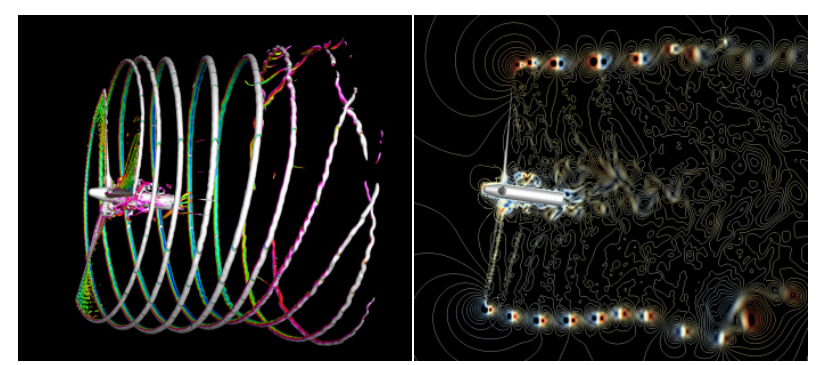

b) $\mathbf{T}_{1}$

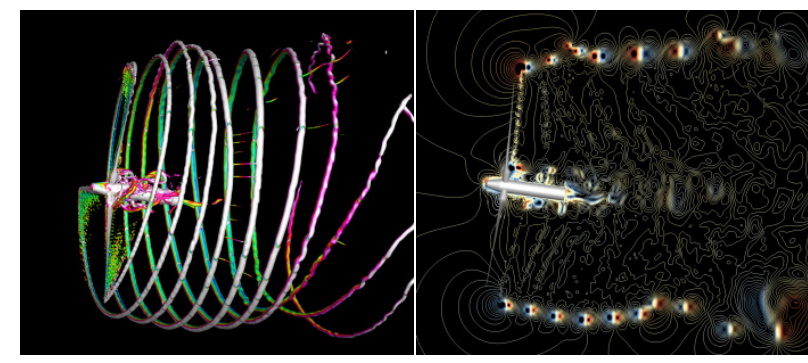

d) $T_{3}$

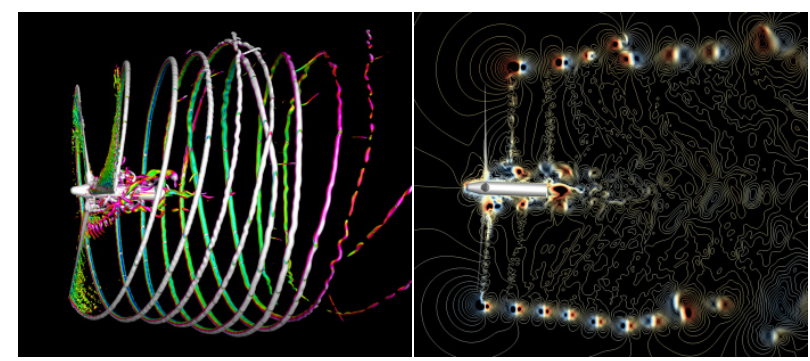

f) $\mathbf{T}_{5}$

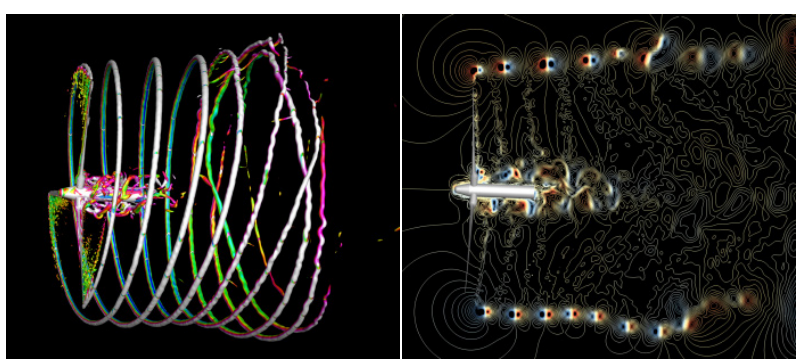

h) $\mathbf{T}_{7}$

Fig. 19 Pitch motion: Q-criterion isosurface (left) and velocity in the symmetry plane (right) at different timesteps for $A_{P I T C H}=4^{\circ}$ 


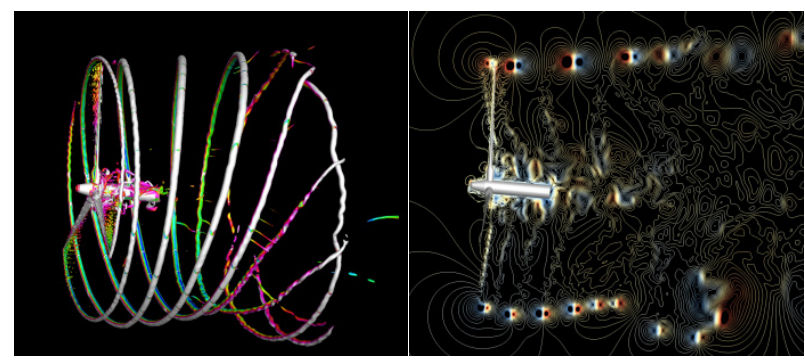

a) $\mathbf{T}_{0}$

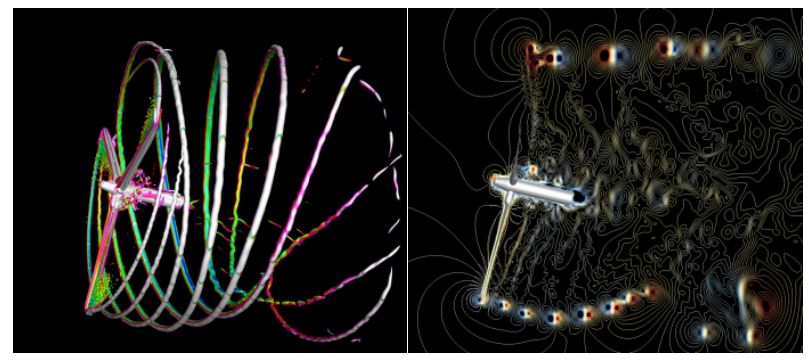

c) $\mathbf{T}_{2}$

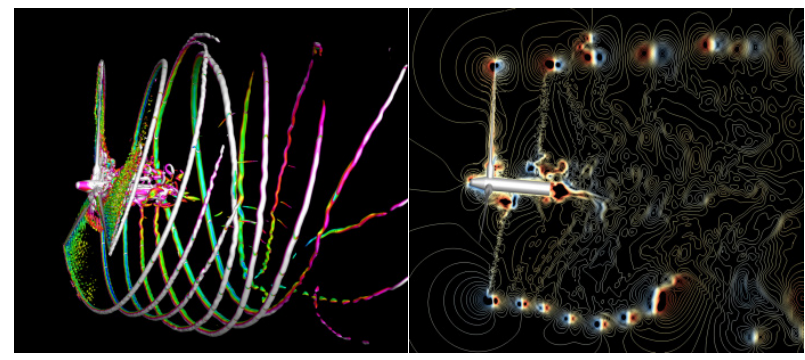

e) $\mathbf{T}_{4}$

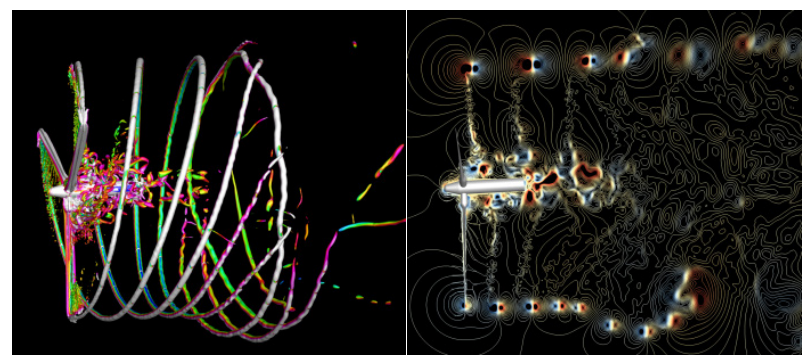

g) $T_{6}$

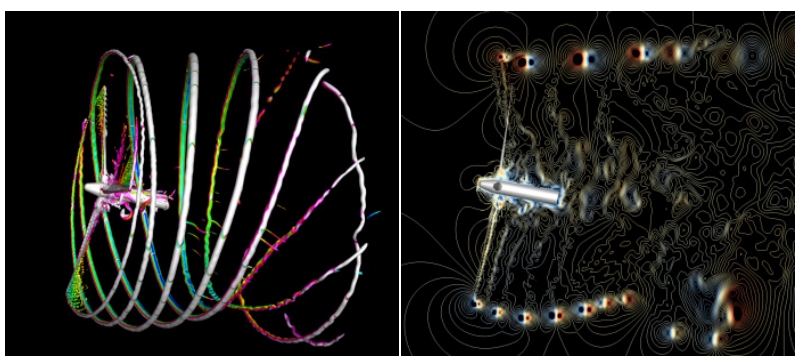

b) $\mathrm{T}_{1}$

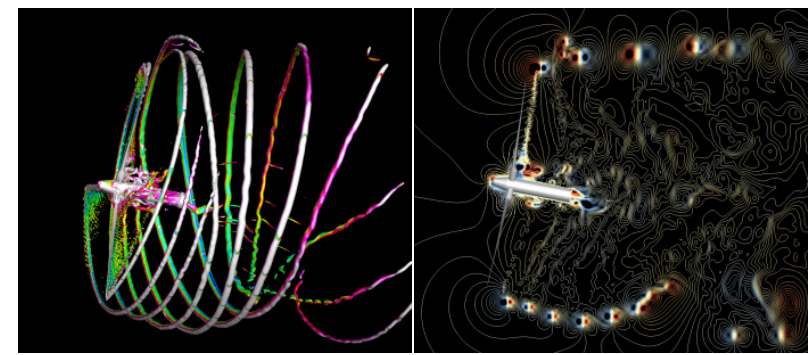

d) $\mathbf{T}_{3}$

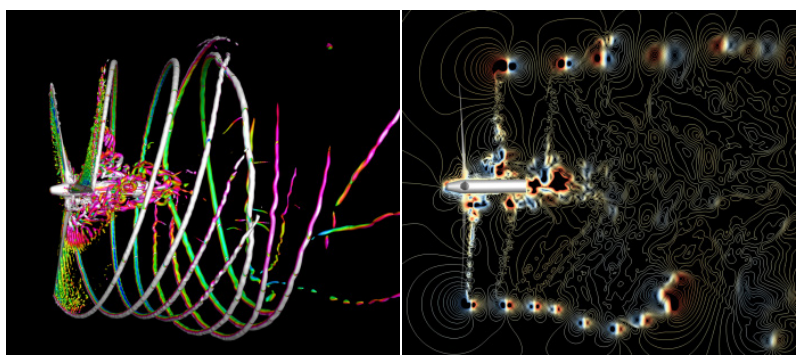

f) $\mathbf{T}_{5}$

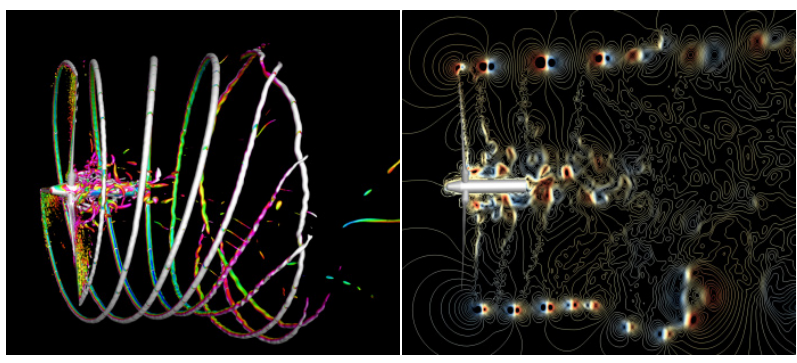

h) $\mathbf{T}_{7}$

Fig. 20 Pitch motion: Q-criterion isosurface (left) and velocity in the symmetry plane (right) at different timesteps for $A_{P I T C H}=8^{\circ}$ 


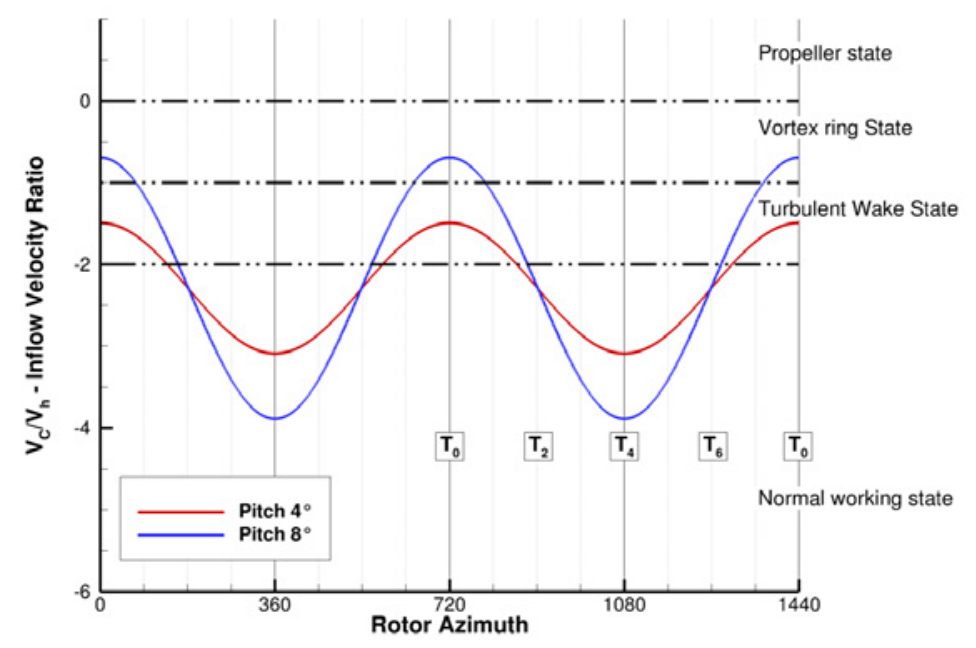

Fig. 21 Pitch motion: VRS identification scheme

\section{Simulation of the floating offshore wind turbine: surge motion}

\section{A. Motion description}

The surge motion of the FOWT in elsA computations also consists in a prescribed motion applied to the whole wind turbine grids (except the background grids), defined as follows:

$$
d x(t)=A_{\text {SURGE }} * \sin \left(2 \pi f_{\text {SURGE }} t\right)
$$

$A_{S U R G E}$ refers to the surge motion amplitude and $f_{S U R G E}$ to the surge motion frequency. Two surge amplitudes have been simulated $(8 \mathrm{~m}$ and $16 \mathrm{~m}$ ) with the same constant frequency compared to the pitch cases (ie. half the rotor frequency).

Fig. 22 is a representation of the surge motion (for $A_{S U R G E}=8 \mathrm{~m}$ ) and should help to analyze the upcoming results. The wind turbine axial displacement is plotted over two surge periods in red (a positive $d x$ corresponding to a backward position), as well as the displacement velocity in green. Comparably to what happens for a pitch motion, the maximum displacement velocity in absolute terms is obtained when the wind turbine comes back to its initial position ( $\mathrm{T}_{0}$ and $\mathrm{T}_{4}$, corresponding to $d x(t)=0$ ). The inflow velocity normal to the rotor plane (at the hub center) can be defined with the following equation and is added in grey:

$$
V_{\text {norm }}=V_{W I N D}-d \dot{x}
$$

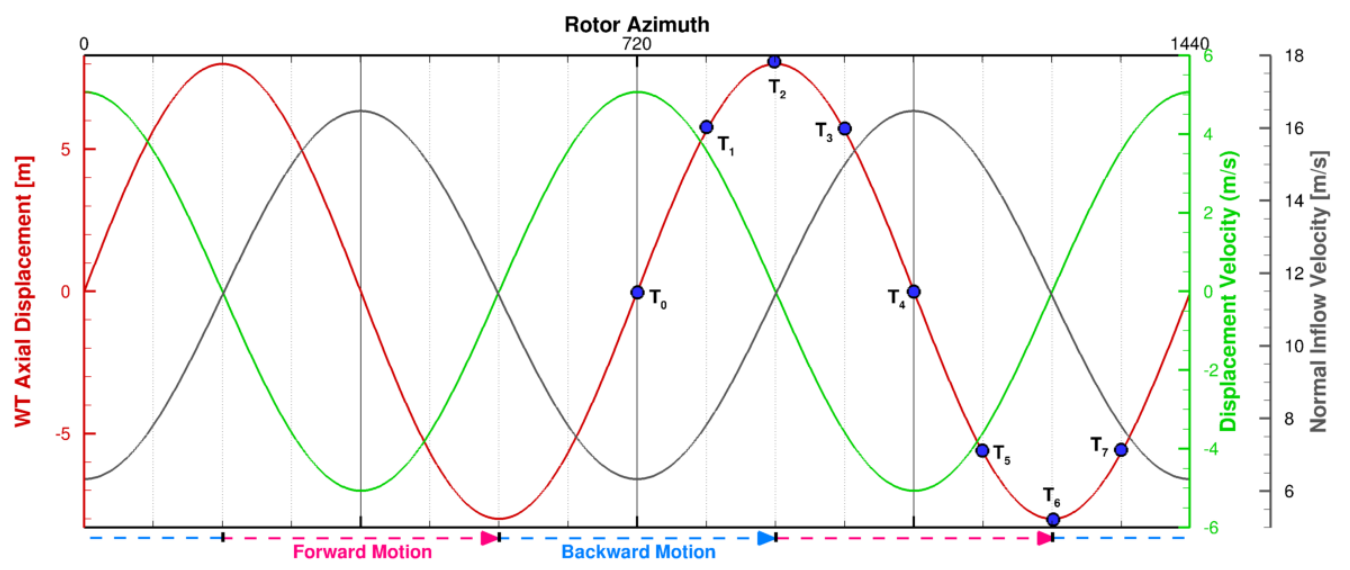

Fig. 22 Surge motion analysis (for $A_{\text {SURGE }}=8 \mathrm{~m}$ and $f_{\text {SURGE }} \approx 0.1 \mathrm{~Hz}$ ): WT axial displacement (red), WT displacement velocity (green) and relative axial velocity (grey) 
The maximum normal inflow velocity is obtained at $\mathrm{T}_{4}$, when the wind turbine comes back to its initial position in a forward motion.

\section{B. Wind turbine loads}

As already done for the pitch cases, the wind turbine global thrust and power are here represented as function of time (Fig. 23) and as function of the axial displacement (Fig. 24). Despite large variations on the global thrust and power, the same behavior is observed compared to the pitch motion: the mean thrust is only slightly decreased compared to the fixed WT case, whereas the mean power is greatly impacted $\left(+14.8 \%\right.$ for $A_{S U R G E}=8 m,+56.6 \%$ for a $16 \mathrm{~m}$ surge amplitude). The instantaneous power varies from 0MW to $13 \mathrm{MW}$ for the $8 \mathrm{~m}$ surge motion. The $16 \mathrm{~m}$ surge motion is probably excessive compared to what can happen on a real wind turbine, especially when looking at the power variations (from -0.6MW to 23.6 MW): the idea is once again to accentuate (and confirm) the unsteady phenomena which can be observed for $A_{S U R G E}=8 \mathrm{~m}$. Thrust and power maxima and minima are respectively obtained at $\mathrm{T}_{4}$ and $\mathrm{T}_{0}$, which respectively correspond to the maximum and minimum normal inflow velocity.

The hysteresis loops presented in Fig. 24 lead to identical conclusions compared to the pitch motion: for both amplitudes, $\Delta P+>\Delta P-$ and $\Delta T+<\Delta T$-, with a power loop even more flattened in its lower part and sharpen in its upper part. For $A_{S U R G E}=16 \mathrm{~m}$, negative thrust and negative power are obtained at $\mathrm{T}_{0}$. Strong interactions between the wind turbine and its wake should therefore be expected when looking at the instantaneous flowfield.
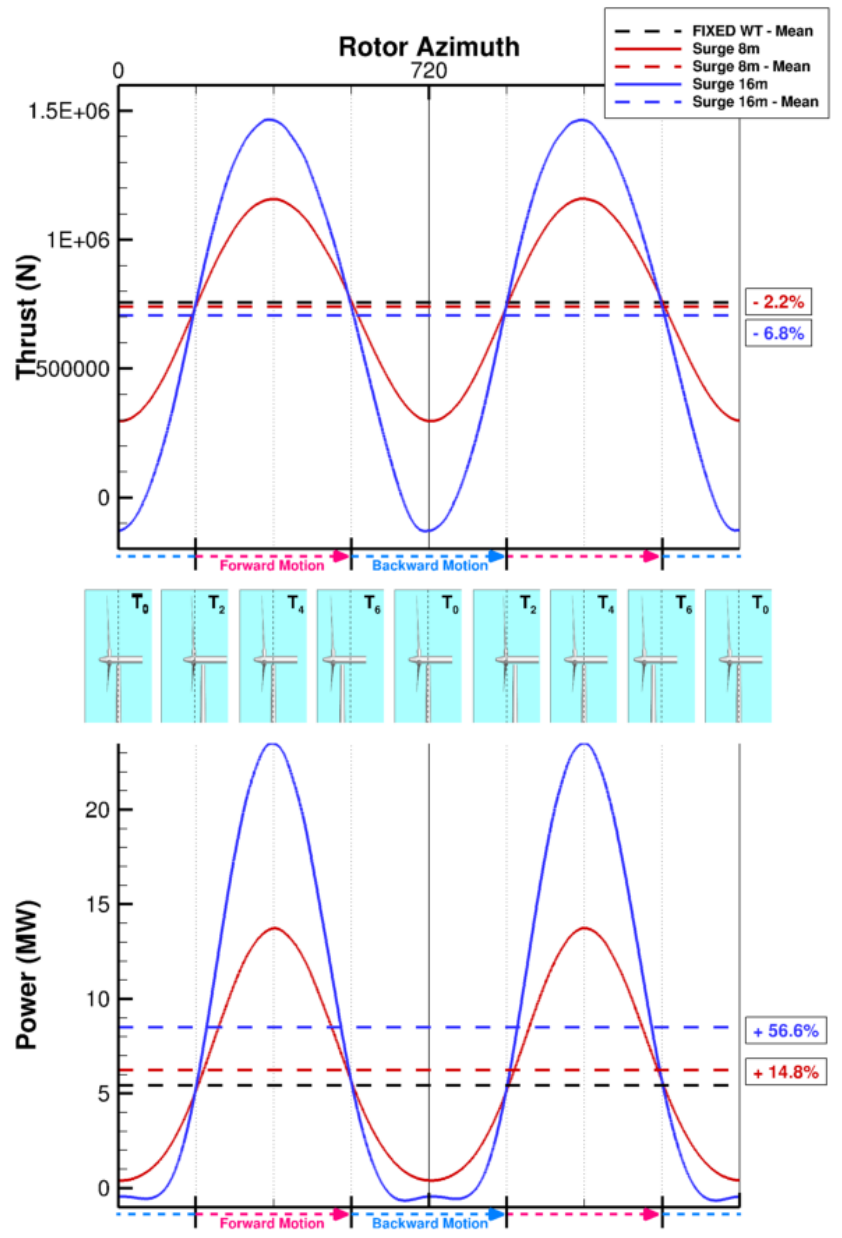

Fig. 23 Surge motion: Global thrust and power as function of time
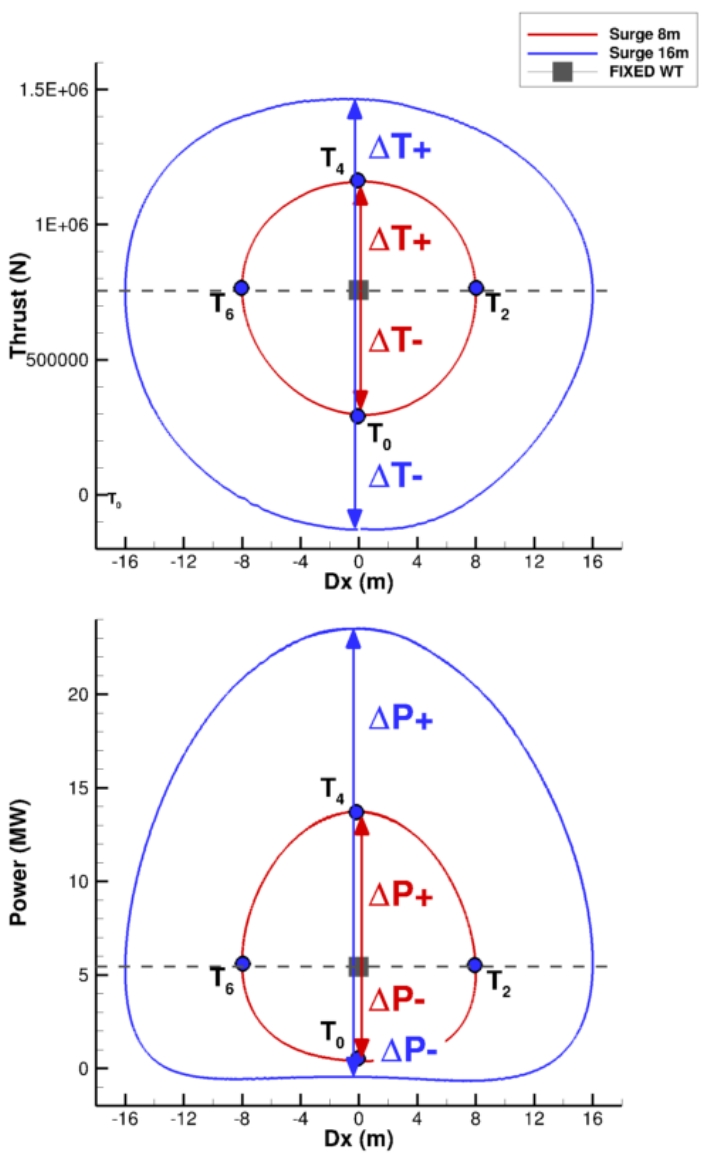

Fig. 24 Surge motion: Global thrust and power as function of pitch angle 
The aerodynamic power produced by each blade during the surge motion is presented in Fig. 25. Since the surge motion consists in a pure translation, the power curves are almost identical for the three blades: the maximum power is reached at $\mathrm{T}_{4}$ and the minimum at $\mathrm{T}_{0}$.

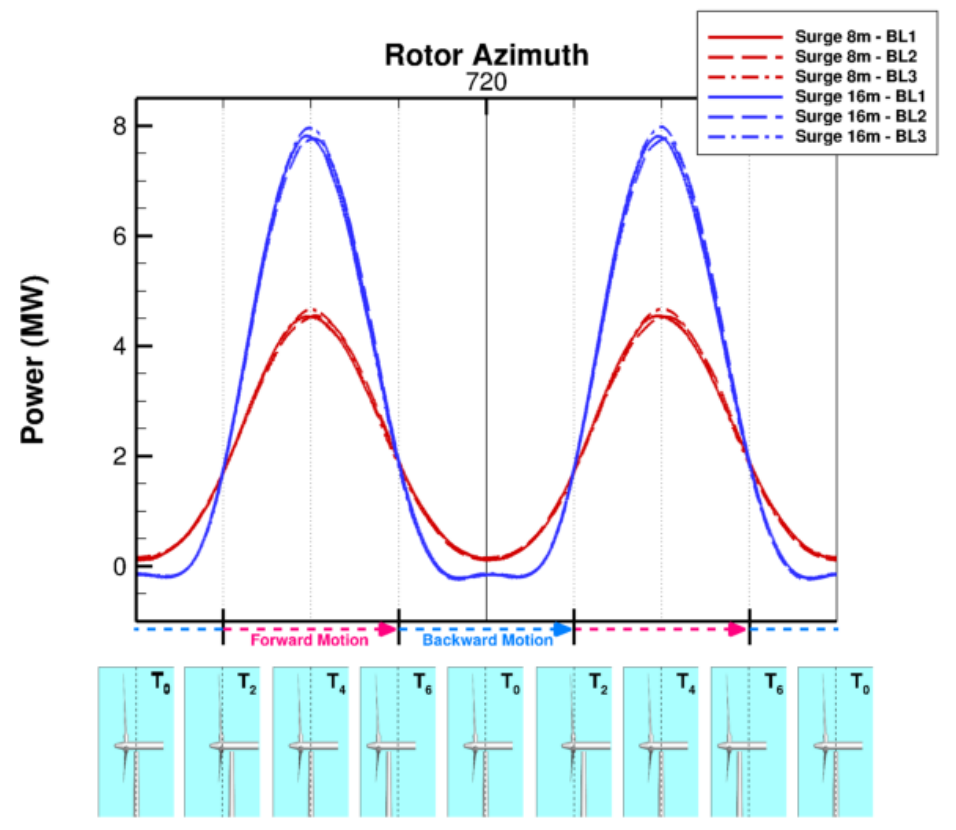

Fig. 25 Surge motion: Blade power variations as function of time

Since the integrated loads are identical on all the blades, it is expected to have identical PUMA results in terms of sectional loads, of local blade section velocity variations due to the surge motion and of local angle of attack.

At a given time, it is actually noticeable in Fig. 26 and Fig. 27 that the three blades see an identical inflow (same velocity magnitude and same angle of attack). Neglecting the small effect of the shaft angle, the incoming flow is indeed axisymmetric, which was not the case in pitch motion.

Comparably to what happened during the pitch motion, the motion has only little impact on the local blade section velocity magnitude (Fig. 26) but rather on the local angle of attack (Fig. 27).

These maps extracted from PUMA simulations are however interesting to develop a deeper understanding of the wake effect on the wind turbine. Let's compare for example the results obtained on blade 1 at $T_{2}$ and $T_{6}$, which correspond to the extremum displacements of the wind turbine, respectively downstream and upstream the central position (highlighted in red in Fig. 26 and Fig. 27). At these positions, the displacement velocity is equal to zero (cf. green curve in Fig. 22) so the wind turbine is supposed to see an identical incoming flow. The obtained local velocity magnitude and angle of attack are nevertheless different, due to the wake induced velocities. At $T_{2}$, the wind turbine is very close to its own wake, producing higher induced velocities on the blade, decreasing the local blade section velocity and angle of attack. At $T_{6}$, the wind turbine is further away from its wake, so the local velocity and angle of attack are higher. It should however be kept in mind that this effect may partially be overestimated in these PUMA simulations, because of the free-wake approach itself (no dissipation of the vortices). 

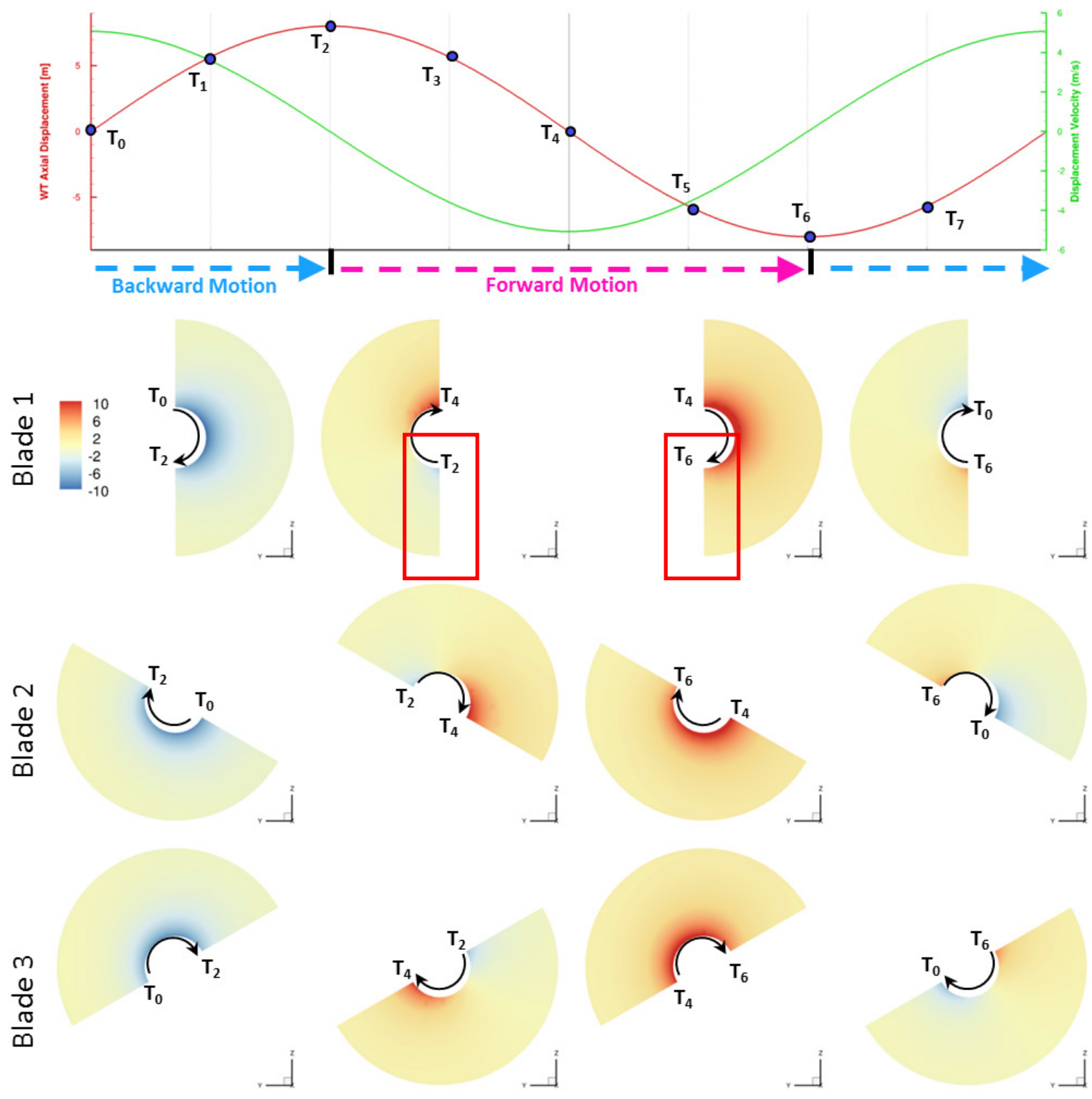

Fig. 26 Surge motion: Local blade section velocity magnitude variations due to the surge motion with $A_{\text {SURGE }}=8 m$ (in \%) 

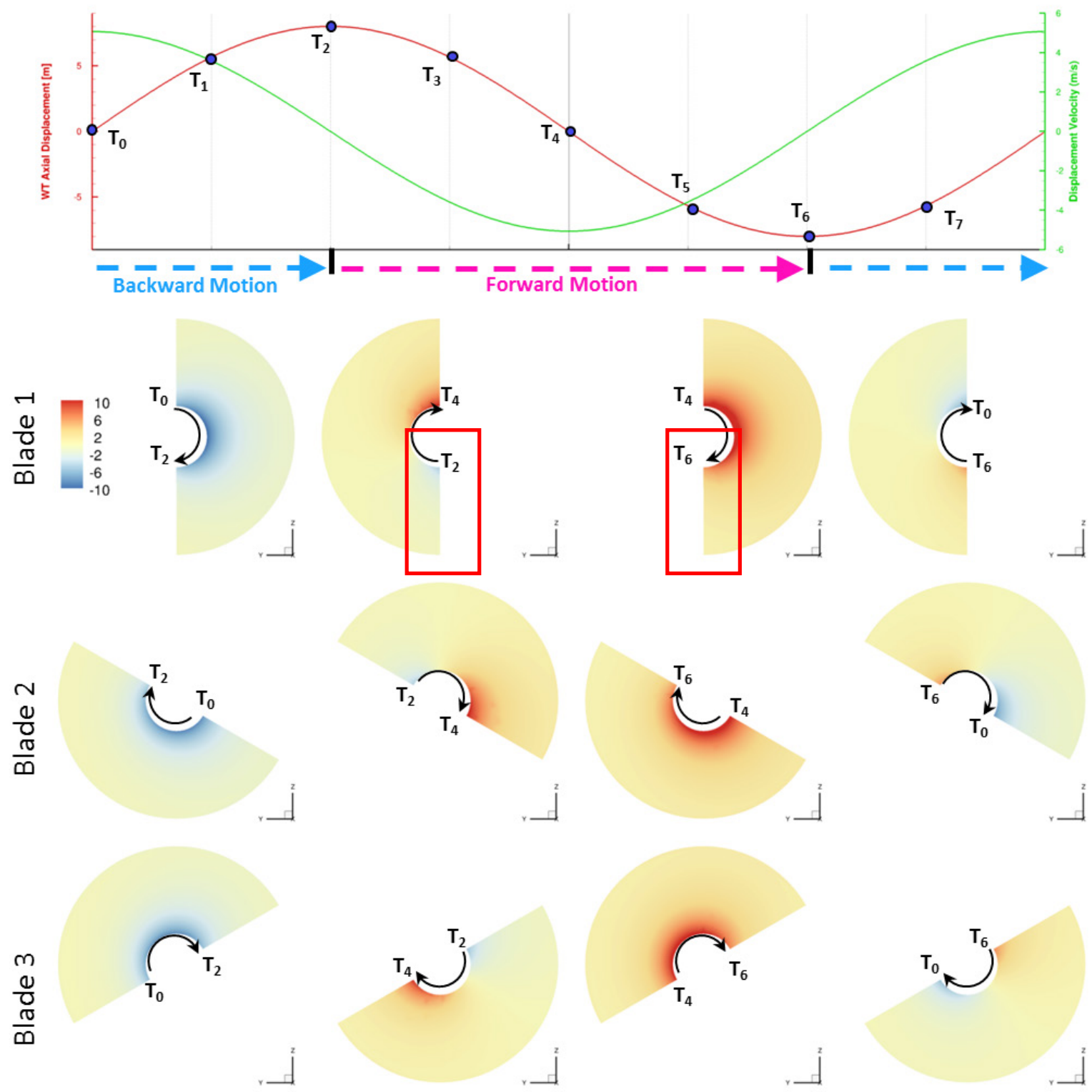

Fig. 27 Surge motion: Local blade section angle of attack for the 8m-amplitude test case

\section{Blade spanwise loads}

The mean spanwise aerodynamic power is shown in Fig. 28a) for the two surge amplitudes and is identical on the three blades. The spanwise power envelope obtained on each blade for $A_{S U R G E}=8 \mathrm{~m}$ is almost the same as well. For this surge amplitude, the obtained aerodynamic power remains always positive, which is coherent with what was observed in Fig. 25. 

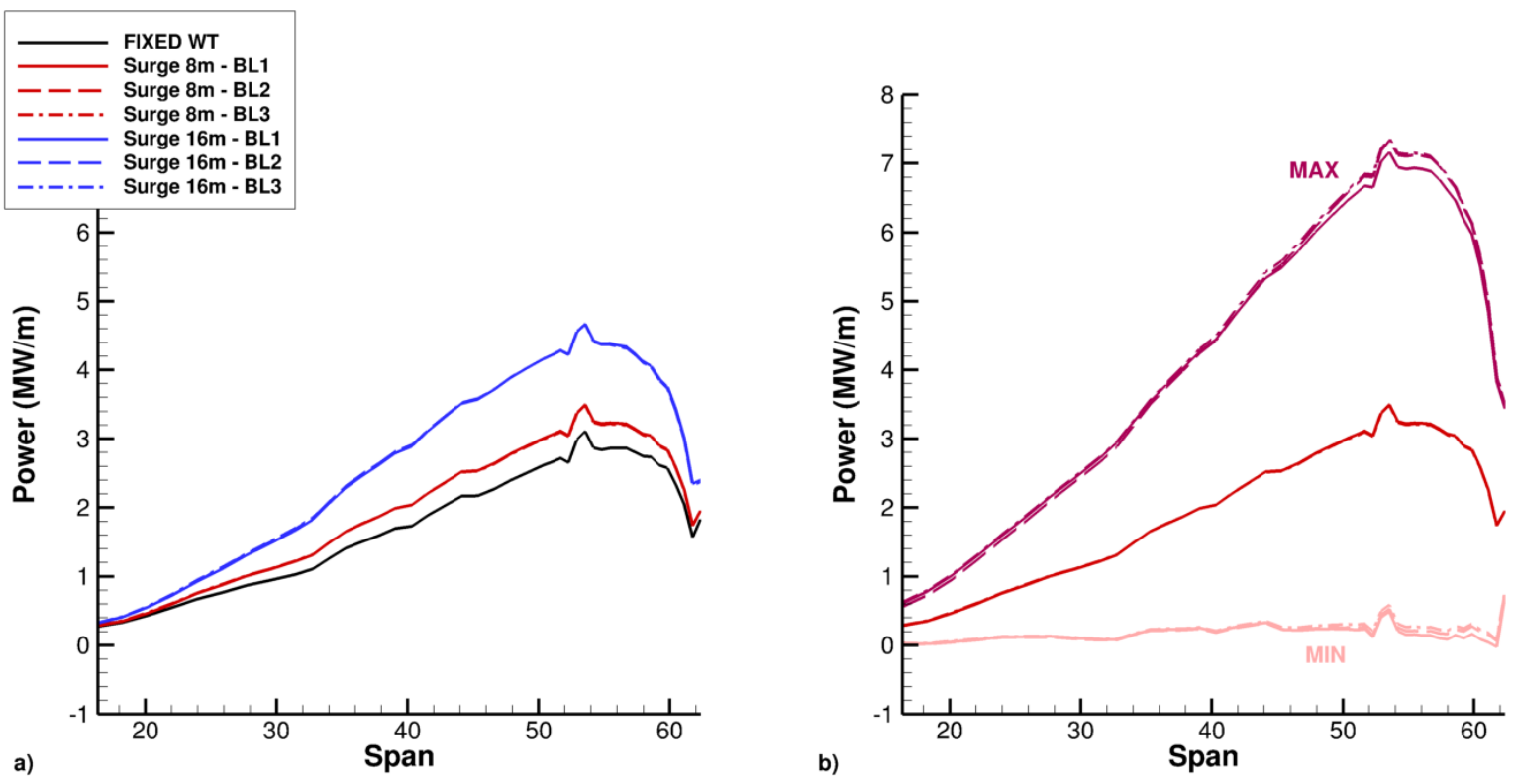

Fig. 28 Surge motion: a) Mean spanwise power on the 3 blades compared to the fixed WT case; b) Mean spanwise power and power envelope on the 3 blades for $A_{S U R G E}=8 \mathrm{~m}$

\section{Wake visualizations}

Finally, the wake of the wind turbine at the identified timesteps $T_{0}, T_{1}, T_{2}, \ldots$ is presented for both surge amplitudes in Fig. 29 and Fig. 30, and the "helicopter-like" VRS scheme in Fig. 31. It is reminded that a positive axial displacement corresponds to a downstream position. The wakes are once again quite well convected and not too much dissipated: several interactions phenomena can be observed.

First, it can be noticed that the surge motion with $A_{S U R G E}=8 \mathrm{~m}$ is quite equivalent in terms of normal inflow velocity at the wind turbine nacelle to the pitch motion with $A_{P I T C H}=4^{\circ}$ (Fig. 9 vs. Fig. 22). Less aerodynamic interactions are yet visible for the surge case (for example Fig. 19a) vs. Fig. 29a)). This is due to the fact that the surge motion is a pure translation: the wind turbine remains a little bit further from its wake since it is not inclined, and the displacement velocity at its top is the same as at its center, thus a little bit lower compared to the pitch motion.

It seems that the FOWT experiencing 8m-amplitude surge motion does not enter a VRS regime. At $\mathrm{T}_{0}$, the tip vortex is emitted very close to the previous one but the blade is not immersed in the wake (Fig. 29a)). This observation is in agreement with Fig. 31.

On the other hand, interactions become significant for $A_{S U R G E}=16 \mathrm{~m}$ and the wind turbine experience severe VRS conditions. Fig. 30 even highlights that at $T_{0}$, when blade 1 crosses the symmetry plane, its tip vortex is emitted almost in the core of the previous one and immediately interacts with it. Indeed, when comparing the top of the wake at T0 and T1 (Fig. 30a) and b)), the same number of vortices is visible (because of blade 1 "missing" vortex). This is also confirmed when looking at Fig. 32 which is a zoomed view obtained at $\mathrm{T}_{0}$ and $\mathrm{T}_{0}+6^{\circ}$. Afterward, blade 2 tip vortex is emitted very close to this "double-vortex" (as seen at T2 in Fig. 30c)), leading to vortex pairing and this pair is convected faster in the wake compared to the other vortices (cf. Fig. 30d), e) and f)). Vortex meandering quickly appears in the wake, leading to significant flow instabilities behind the FOWT.

The FOWT undergoing the $16 \mathrm{~m}$-amplitude surge motion actually probably switches to a Propeller state around $\mathrm{T}_{0}$ (both thrust and power seen in Fig. 23 are negative). The curve from Fig. 31 does not outline it because of some limitations of the helicopter approach adopted to produce it: the mean thrust over one motion surge is used to estimate the "theoretical" hover induced velocity, keeping the ratio $V_{c} / V_{h}$ from reaching zero with non zero axial velocities. Moreover, $V_{h}$ is estimated with $V_{h}=\sqrt{T / 2 \rho S}$ which is no longer valid for negative thrust. This representation is not an absolute criterion for identifying Vortex Ring State or Propeller State, it rather consists in an indicator which has to be confirmed by wake visualizations. 
Eventually, it is also more visible on this surge case that the blade wake emitted for a backward motion of the wind turbine is greatly distorted on at least one-third of the blade (Fig. 29c) and d), more visible in Fig. 30c) and d)). This wake distortion is linked to the negative thrust which is obtained on this blade portion when the wind turbine is moving faster backward.

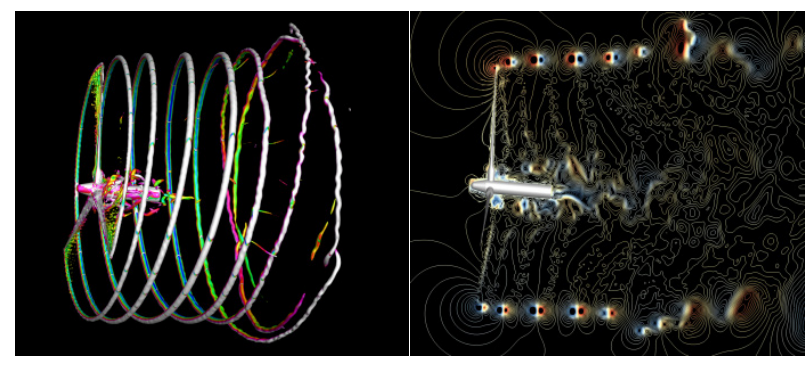

a) $\mathbf{T}_{\mathbf{0}}$

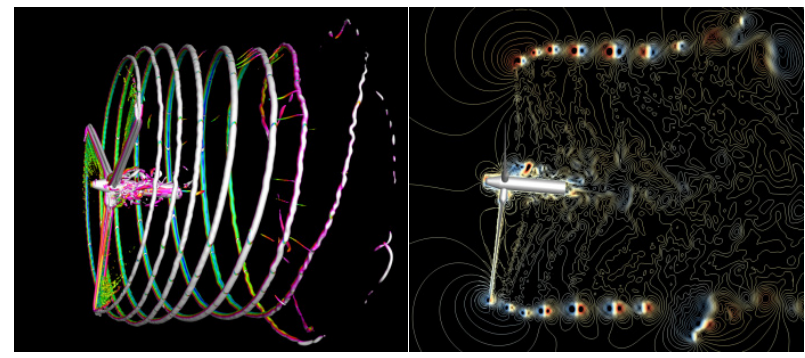

c) $\mathbf{T}_{2}$

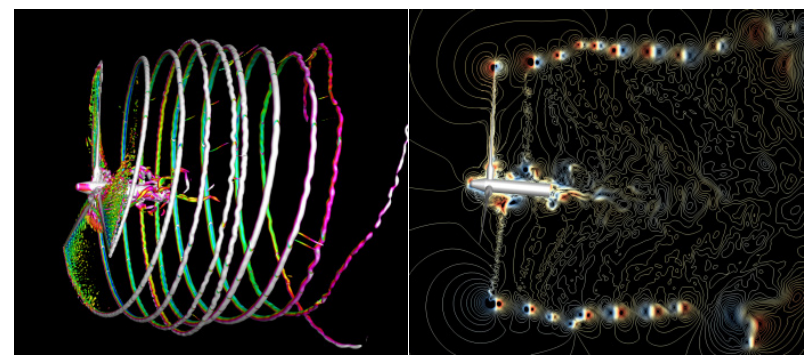

e) $\mathbf{T}_{4}$

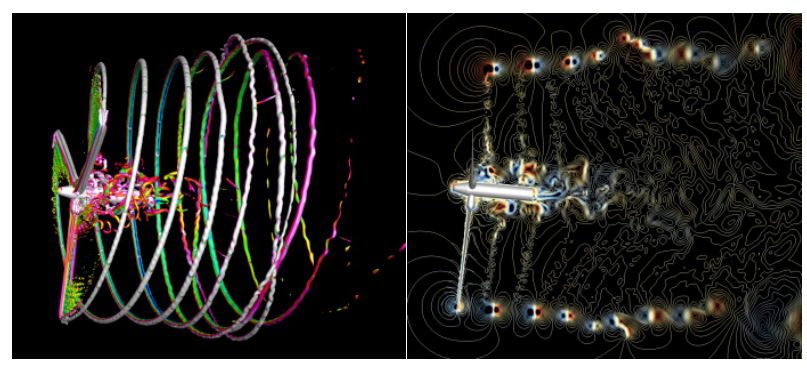

g) $T_{6}$

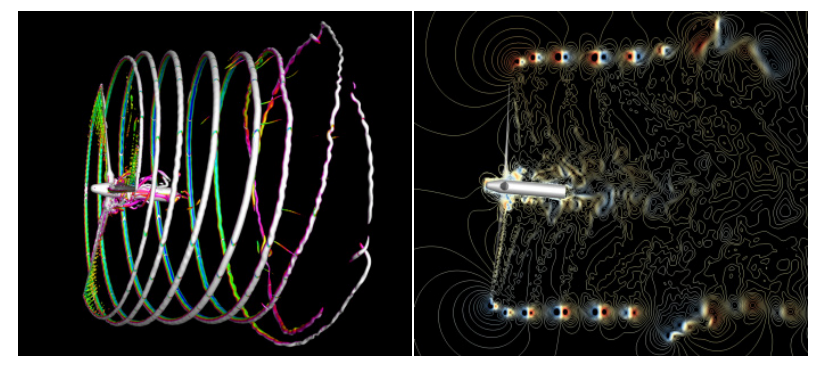

b) $T_{1}$

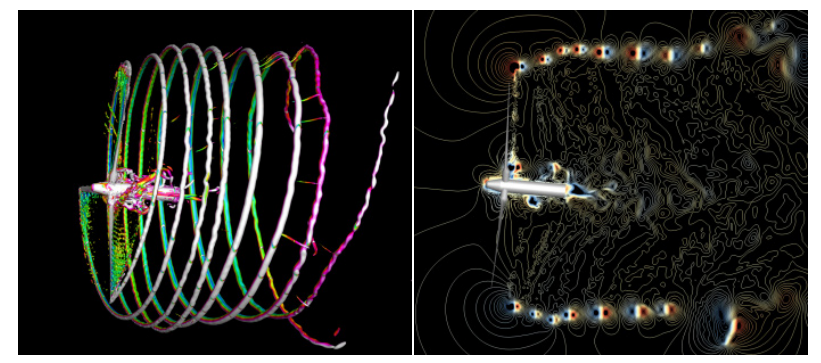

d) $T_{3}$

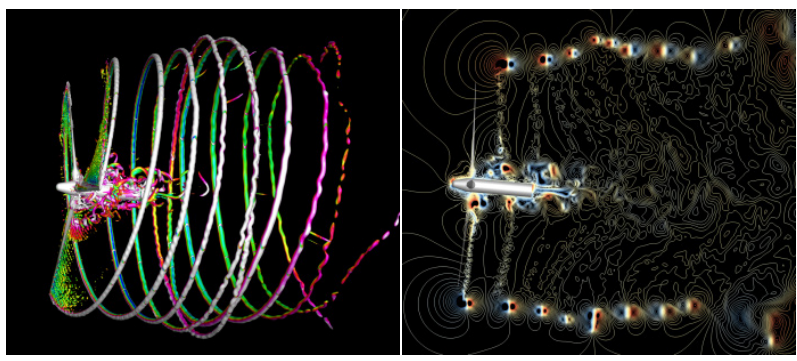

f) $\mathbf{T}_{5}$

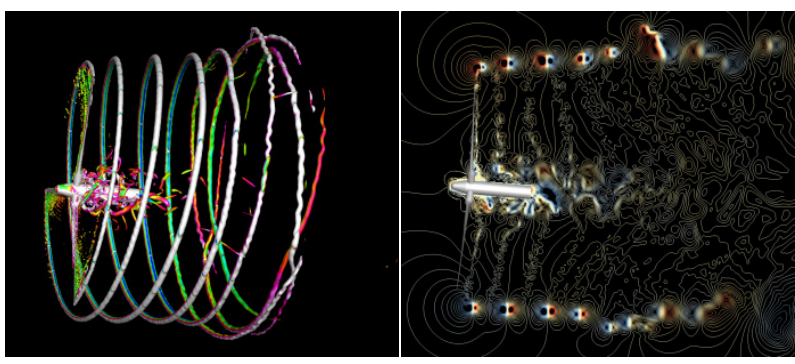

h) $\mathbf{T}_{7}$

Fig. 29 Surge motion: Q-criterion isosurface (left) and velocity in the symmetry plane (right) at different timesteps for $A_{S U R G E}=8 m$ 


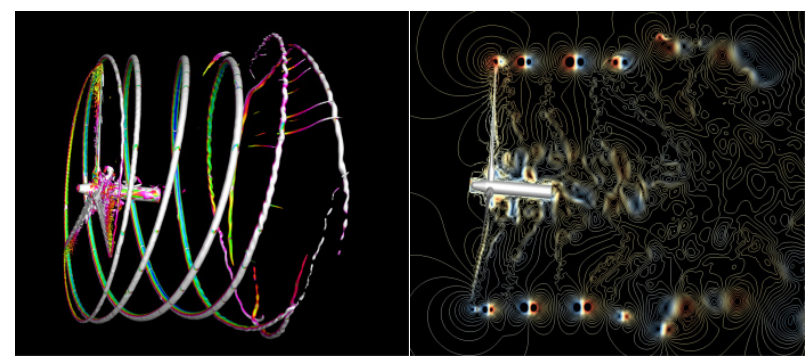

a) $\mathbf{T}_{0}$

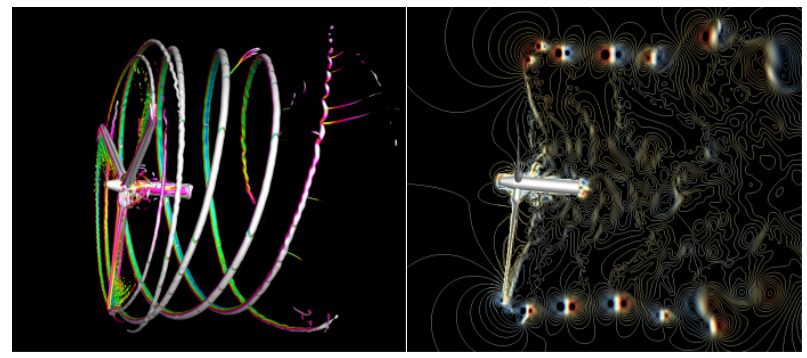

c) $T_{2}$

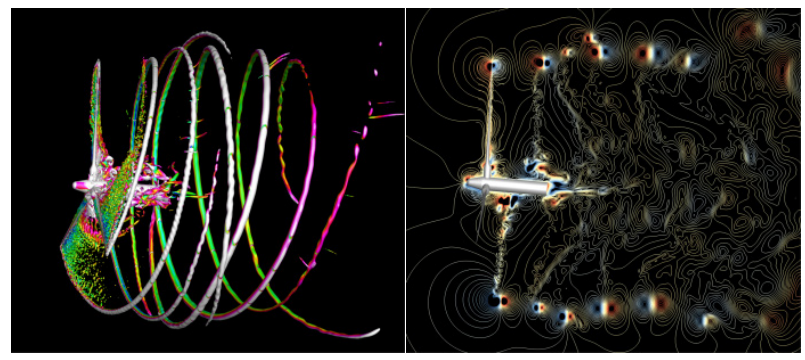

e) $\mathbf{T}_{4}$

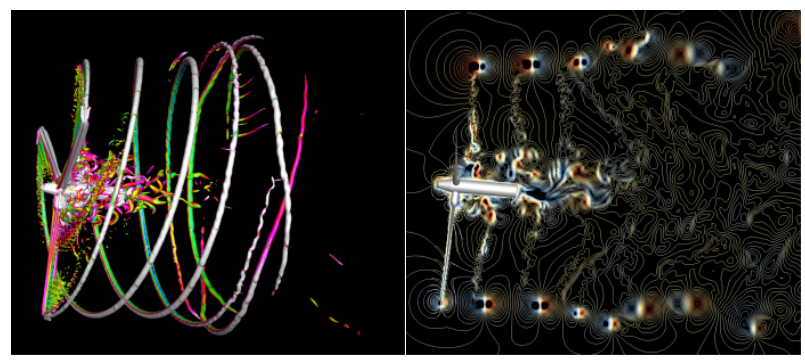

g) $T_{6}$

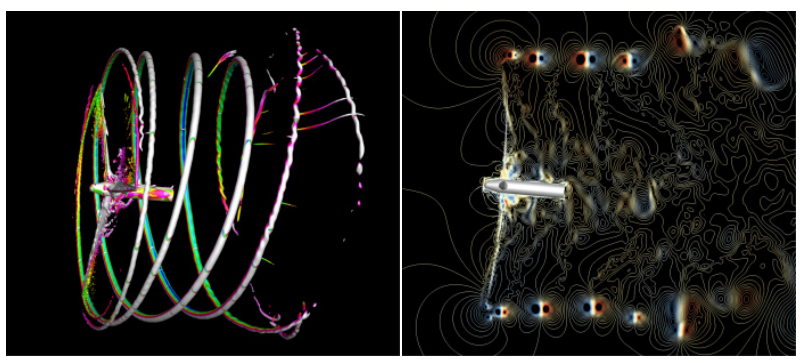

b) $\mathrm{T}_{1}$

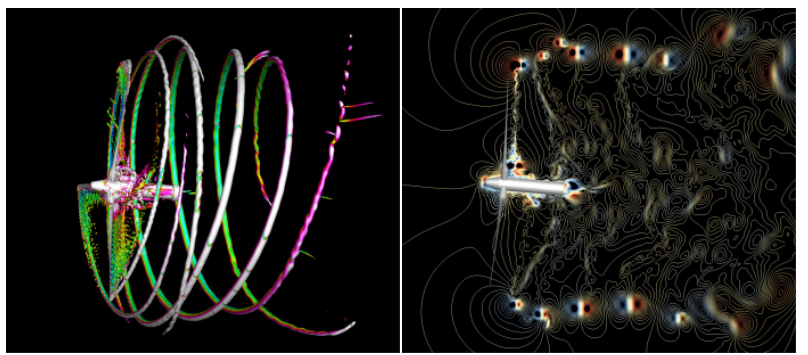

d) $\mathbf{T}_{3}$

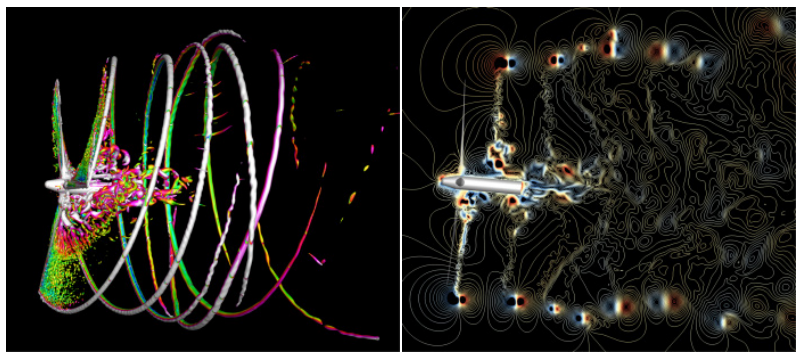

f) $\mathbf{T}_{5}$

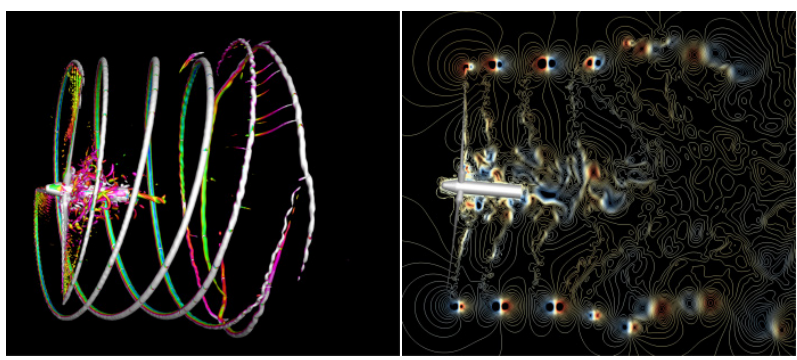

h) $\mathbf{T}_{7}$

Fig. 30 Surge motion: Q-criterion isosurface (left) and velocity in the symmetry plane (right) at different timesteps for $A_{S U R G E}=16 \mathrm{~m}$ 


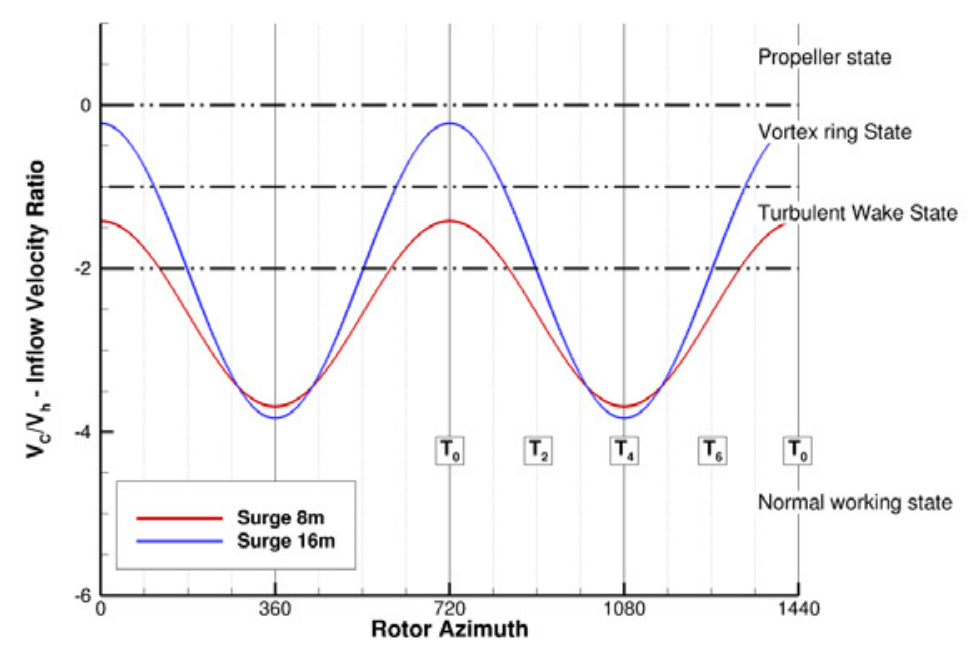

Fig. 31 Surge motion: VRS identification scheme
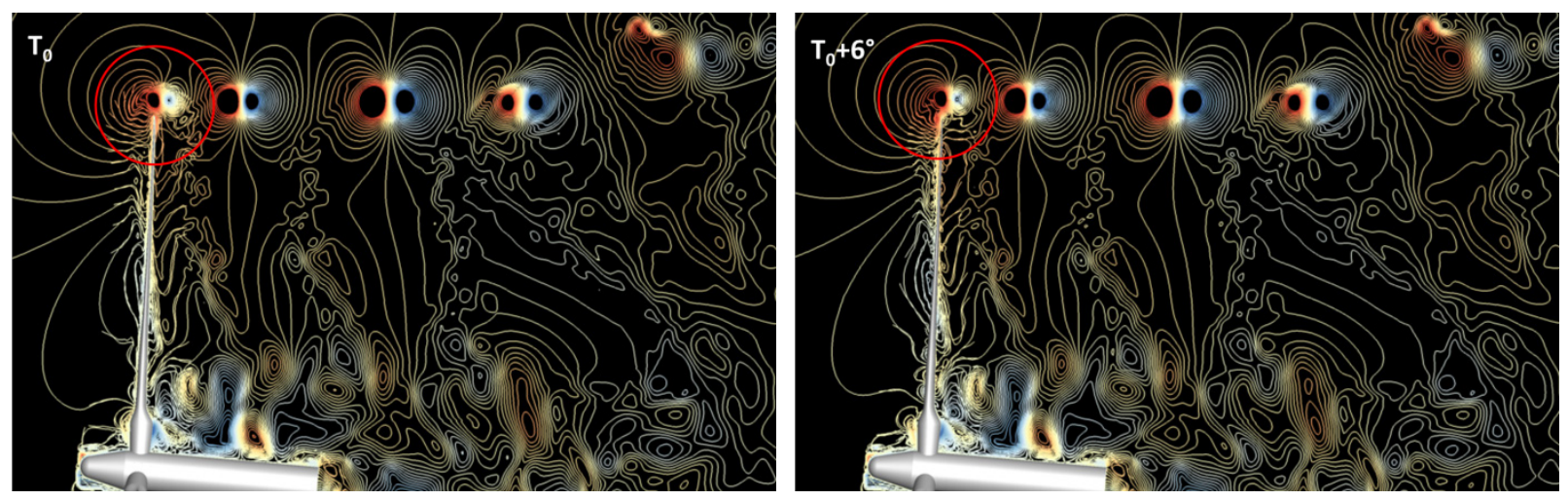

Fig. 32 Surge motion for $A_{S U R G E}=16 \mathrm{~m}:$ zoomed view at $\mathrm{T}_{0}$ and $\mathrm{T}_{0}+6^{\circ}$

\section{Conclusions}

The aerodynamic behavior of a wind turbine is already quite complex to investigate, due to several unsteady phenomena that can occur (unsteady inflow, yaw conditions, dynamic stall,...). This is all the more true when investigating the flowfield around a floating offshore wind turbine which is subject to the ocean waves. It has been demonstrated in the literature that, among the 6 degrees of freedom involved by the waves, two of them could have a significant impact on the wind turbine aerodynamic: pitch and surge motions.

The current paper therefore investigated the effect of these two prescribed motions on aerodynamic performance and on the wind turbine wake thanks to CFD simulations. For each motion type, several amplitudes have been tested.

It turns out that both pitch and surge motions have a similar effect on the wind turbine global loads: the mean thrust is only slightly impacted (from $1 \%$ to $7 \%$ decrease) whereas the mean aerodynamic power is greatly increased (from 10\% to 57\%). The power gain is obtained during the forward motion of the wind turbine (pitch or surge) and reaches its maximum when the wind turbine is back to its central position during this forward phase (maximum angular/displacement velocity). The instantaneous loads variations on the blade are consequent as well, and can differ from one blade to the others in the case of pitch motion.

Theses loads variations are due to significant variations of the blade sections angle of attack. The local velocity magnitude is indeed only slightly modified, since it is indeed dominated by the rotational velocity on such configurations.

The loads variations and the effect of both motions on the wind turbine may however be overestimated due to the modeling itself: blade pitch controller was not considered in this investigation and the rotation speed was prescribed 
in all simulations. The waves frequencies are probably too high to consider limiting their effect on performance with blade pitch control. The fixed and prescribed rotation speed in both PUMA and elsA codes, though, is a limitation on the presented computations.

Furthermore, it has been demonstrated that vortex ring state (VRS) (as observed for helicopters in descending flight) could be encountered by the FOWT for the highest considered amplitudes. This obviously occurs when the wind turbine is quickly moving backward. Even for the cases where VRS is not reached, strong interactions have been noticed between the blades and the wake, leading to vortex pairing, wake meandering and instabilities.

If PUMA results (free wake method) have been punctually used to complement the present analysis, the occurrence of all these complex unsteady aerodynamic phenomena underline all the benefits of using CFD to accurately predict the aerodynamic performances and behavior of FOWT.

\section{Acknowledgments}

Most of this work was granted access to the HPC resources of CINES under the allocation 2018 - A0032A10280 made by GENCI.

\section{References}

[1] Sebastian, T., Lackner, M. A., "Characterization of the unsteady aerodynamics of offshore floating wind turbines”, Wind Energy, Vol. 16, Issue 3, 2013, pp. 339-352

[2] Tran, T. T., Kim, D. H., Song, J., "Computational fluid dynamic analysis of a floating offshore wind turbine experiencing platform pitching motion”, Energies, Vol. 7, 2014, pp. 5011-5026

[3] Tran, T. T., Kim, D. H., "A CFD study into the influence of unsteady aerodynamic interference on wind turbine surge motion”, Renewable Energy, Vol. 90, 2016, pp. 204-228

[4] Tran, T. T., Kim, D. H., “The aerodynamic interference effects of a floating offshore wind turbine experiencing platform pitching and yawing motions”, Journal of Mechanical Science and Technology, Vol. 29 (2), 2015, pp. 549-561

[5] Leble, V., Barakos, G., "Rigid Actuation of the Wind Turbine”, $72^{\text {nd }}$ Annual Forum of the American Helicopter Society, Palm Beach, FL, USA, 17-19 May 2016, ISBN 9781510825062

[6] Bekiropoulos, D., et al., "Simulation of unsteady aerodynamic effects on floating offshore wind turbines", $11^{\text {th }}$ German Wind Energy Conference, 2012

[7] Cheng, P., Wan, D., Hu, C., "Unsteady Aerodynamic Simulations of Floating Offshore Wind Turbines with Overset Grid Technology”, $26^{\text {th }}$ International Ocean and Polar Engineering Conference, 2016

[8] Li, P., et al., "Numerical simulations of wake flows of floating offshore wind turbines by unsteady actuator line model”, Proceedings of the $9^{\text {th }}$ International Workshop on Ship and Marine Hydrodynamics, 2015

[9] Courbois, A., "Etude expérimentale du comportement dynamique d'une éolienne offshore flottante soumise à l'action conjuguée de la houle et du vent”, PhD Dissertation, Ecole Centrale de Nantes, 2013

[10] Lienard, C., Boisard, R., "Investigation of the MEXICO rotor aerodynamics in axial flow, including boundary layer transition effects”, 2018 Wind Energy Symposium, AIAA SciTech Forum, Kissimmee, FL, USA, 8-12 January 2018

[11] Jonkman, J., Butterfield, S., Musial, W., Scott, G., “Definition of a 5MW Reference Wind Turbine for Offshore System Development”, Technical Report NREL/TP-500-38060, National Renewable Energy Laboratory, Golde, CO, USA, 2009

[12] Sebastian, T., “The aerodynamic and near wake of an offshore floating horizontal axis wind turbine”, PhD Dissertation, University of Massachusetts, Amherst, MA, USA, 2012

[13] Mudry, M., “La théorie des nappes tourbillonnaires et ses applications à l'aérodynamique instationnaire”, PhD Dissertation, Paris VI University, 1982

[14] R. Boisard, "Aerodynamic investigation of a helicopter rotor hovering in the vicinity of a building", 74th AHS forum, Phoenix, Arizona, USA, 14-17 May 2018

[15] Cambier L., Veuillot J.-P., "Status of the elsA CFD software for Flow Simulation and Multidisciplinary Applications”, 46 ${ }^{\text {th }}$ AIAA Aerospace Science Meeting and Exhibit, AIAA Paper 2008-664, Reno, Nevada, USA, January 2008

[16] Cambier, L., Heib, S., Plot, S., “The ONERA elsA CFD software: input from research and feedback from industry”, Mechanics and Industry, 14 (3), 2013, pp. 159-174 
[17] Benoit, C., Peron, S., Landier, S., "Cassiopee : a CFD pre- and post-processing tool", Aerospace Science and Technology, vol. 45, pp. 243-272, 2015

[18] Kirby, A., Brazell, M., Yang, Z., Rajib, R., Ahrabi, B., Mavriplis, D., Sitaraman, J., Stoellinger, M., "Wind farm simulations using an overset hp-adaptive approach with blade-resolved turbine models”, $23^{\text {rd }}$ Aerospace Sciences Meeting and Exhibit, Aerospace Sciences Meeting, AIAA Paper 2017-3958, Denver, CO, Jun. 5-7, 2017

[19] Leishman, J.G., "Principles of Helicopter Aerodynamics”, Cambridge University Press, Second Edition, July 2006. 